\title{
Effect of let-7c on the PI3K/Akt/FoxO signaling pathway in hepatocellular carcinoma
}

\author{
YUEHUA LI ${ }^{1}$, PENGFEI LI ${ }^{2}$ and NA WANG ${ }^{1}$ \\ ${ }^{1}$ College of Information and Computer, Taiyuan University of Technology, Taiyuan, Shanxi 030024; \\ ${ }^{2}$ Translational Medicine Research Center, Shanxi Medical University, Taiyuan, Shanxi 030001, P.R. China
}

Received January 30, 2020; Accepted October 19, 2020

DOI: 10.3892/ol.2020.12357

\begin{abstract}
The early diagnosis and treatment of liver hepatocellular carcinoma (LIHC) remains a major challenge. Therefore, it is of great significance to strengthen basic research on LIHC in order to improve the prevention and treatment of the disease. Numerous studies have indicated that the PI3K/Akt and FoxO signaling pathways mediate proliferation, survival and migration during the development of LIHC. Therefore, they have become a target for LIHC treatment. Furthermore, let-7c has been demonstrated to repress cell proliferation, migration and invasion, and to induce $G_{1}$ phase arrest and apoptosis of LIHC cells. However, the mechanism of its action is not clear. In the present study, the association between let-7c and the PI3K/Akt/FoxO signaling pathway, as well as their roles in the development of LIHC were investigated using The Cancer Genome Atlas and various public databases (Tumor-miRNA-Pathway, OncomiR, DIANA-TarBase v8, KOBAS 3.0, ONCOMINE, Kaplan-Meier plotter, LinkedOmics, UALCAN and cBioPortal). The effects of let-7c-5p on PI3K/Akt/FoxO signaling pathway-related target genes were analyzed following overexpression of let-7c-5p in the MHCC-97H cell line via reverse transcription-quantitative PCR, and the let-7c-5p target genes belonging to the $\mathrm{PI} 3 \mathrm{~K} / \mathrm{Akt} / \mathrm{FOXO}$ signaling pathway in LIHC were screened out. GO and KEGG enrichment analyses of these target genes was performed using g:Profiler, gOST. In addition, GeneMANIA and Search Tool for the Retrieval of Interacting Genes/Proteins (STRING) databases were used to determine the gene-gene and protein-protein interaction networks, respectively. The data demonstrated that cyclin B2 (CCNB2), cyclin E2 (CCNE2), cyclin dependent kinase 4 (CDK4), homer scaffold protein 1 (HOMER1), heat shock protein $90 \alpha$ family class A member 1 (HSP90AA1), neuroblastoma RAS viral
\end{abstract}

Correspondence to: Mrs. Yuehua Li, College of Information and Computer, Taiyuan University of Technology, 79 West Street Yingze, Taiyuan, Shanxi 030024, P.R. China

E-mail: sxjkpa@163.com

Key words: hepatocellular carcinoma, let-7c, PI3K/Akt signaling pathway, FoxO signaling pathway oncogene homolog (NRAS), protein phosphatase 2 catalytic subunit $\alpha$ (PPP2CA), protein kinase AMP-activated catalytic subunit $\alpha 2$ (PRKAA2) and Rac family small GTPase 1 (RAC1) may be target genes of let-7c-5p. These genes, particularly CCNE2, were associated with poor overall survival and could be promising candidate biomarkers for disease and poor prognosis in LIHC. Among them, seven genes (CCNE2, CDK4, HSP90AA1, NRAS, PPP2CA, PRKAA2 and RAC1) belonged to the PI3K-Akt signaling pathway and four genes (CCNB2, HOMER1, NRAS and PRKAA2) belonged to the FoxO signaling pathway. The majority of these genes were closely associated with the cell cycle and their elevated expression may aggravate cell cycle disorders. Therefore, let-7c may be considered to be an anti-oncogene of LIHC. The present study may provide novel targets and strategies for the diagnosis and treatment of LIHC.

\section{Introduction}

Liver cancer is the sixth most common type of cancer and the fourth leading cause of cancer-associated mortality worldwide, with $\sim 841,000$ new cases and 782,000 deaths in 2018 (1). The 5 -year survival rate of liver cancer is estimated to be $18 \%$ (2). Liver hepatocellular carcinoma (LIHC) accounts for $75-85 \%$ of primary liver cancers (1) and its overall median survival times is estimated to be 29.8 months (2.48 years) (3), and this has not improved over time (3). Current treatment methods do not ensure effective early diagnosis and treatment of LIHC. Therefore, improving the prevention and treatment of this disease is imperative.

MicroRNAs (miRNAs) are small non-coding sequences with a length of $20 \mathrm{bp}$, which regulate gene expression following transcription. miRNAs control several eukaryotic developmental and cellular processes (4-6). miRNAs usually bind to the 3'-untranslated region of the target mRNAs to cause their degradation. A previous study has demonstrated that the majority of human protein-coding genes contain at least one conserved miRNA binding site (7), whereas a high number of miRNAs can regulate multiple mRNAs (4), suggesting that the biological functions of miRNAs are highly diverse. Therefore, it is not surprising that disorders of miRNAs are often associated with human diseases, including cancer $(8,9)$. Increasing evidence has revealed that miRNAs are closely associated with the abnormal expression of genes regulating 
hepatocyte proliferation, cell cycle, metastasis and apoptosis, which ultimately leads to the development of LIHC $(10,11)$, and these suggests that miRNAs play an important role in the occurrence and development of LIHC, and have potential diagnostic and therapeutic value $(12,13)$. Among them, let-7c has been identified as an anticancer miRNA in LIHC (14). Additional research has indicated that let-7c represses cell proliferation, migration and invasion, and induces $G_{1}$ phase arrest and apoptosis of LIHC cells (15). Furthermore, let-7c can enhance sorafenib-induced apoptosis of LIHC cells by targeting Bcl-xL (16).

The PI3K/Akt signaling pathway is highly mutated and activated in several cancer types, which mediates the proliferation, survival, migration and angiogenesis of these cancer cells (17-19) and has become a target for human cancer treatment. Notably, the PI3K/Akt signaling pathway is associated with LIHC progression, vascular infiltration and metastasis, as well as poor prognosis and a low survival rate in patients with LIHC (20). Furthermore, the FoxO signaling pathway, which is closely associated with the PI3K/Akt signaling pathway, serves an important role in the occurrence and development of hepatocellular carcinoma $(21,22)$.

Therefore, in the present study, the association between let-7c and the PI3K/Akt/FoxO signaling pathway, as well as their roles in the development of LIHC were investigated using The Cancer Genome Atlas (TCGA) and various public databases. The effects of let-7c-5p on PI3K/Akt/FoxO signaling pathway-related target genes were analyzed following overexpression of let-7c-5p in the MHCC-97H cell line. The results may reveal novel targets and strategies for the diagnosis and treatment of LIHC.

\section{Materials and methods}

Clinical significance of let-7c-5p in hepatocellular carcinoma as determined by TCGA analysis. The Cancer Genome Mapping (derived from TCGA) data portal is the largest and most commonly used public resource, providing datasets, such as somatic mutations, gene expression, gene methylation and copy number variation, for thousands of tumor samples (23). In the present study, the let-7c-5p expression profiles of different types of human cancer and adjacent normal tissues were obtained from Tumor-miRNA-Pathway (http://bioinfo.life.hust.edu.cn/miR_path/), an online TCGA data analysis tool that displays miRNA expression levels in 34 different types of tumor, including LIHC, adrenocortical carcinoma (ACC), bladder urothelial carcinoma (BLCA), breast invasive carcinoma (BRCA), cervical squamous cell carcinoma and endocervical adenocarcinoma (CESC), cholangiocarcinoma (CHOL), colon adenocarcinoma (COAD), lymphoid neoplasm diffuse large b-cell lymphoma (DLBC), esophageal carcinoma (ESCA), FFPE pilot phase II FFPE (FPPP), glioblastoma multiforme (GBM), head and neck squamous cell carcinoma (HNSC), kidney chromophobe (KICH), kidney renal clear cell carcinoma (KIRC), kidney renal papillary cell carcinoma (KIRP), acute myeloid leukemia (LAML), brain lower grade glioma (LGG), lung adenocarcinoma (LUAD), lung squamous cell carcinoma (LUSC), mesothelioma (MESO), ovarian serous cystadenocarcinoma (OV), pancreatic adenocarcinoma (PAAD), pheochromocytoma and paraganglioma (PCPG), prostate adenocarcinoma (PRAD), rectum adenocarcinoma (READ), sarcomav (SARC), skin cutaneous melanoma (SKCM), stomach adenocarcinoma (STAD), testicular germ cell tumors (TGCT), thyroid carcinoma (THCA), thymoma (THYM), uterine corpus endometrial carcinoma (UCEC), uterine carcinosarcoma (UCS) and uveal melanoma (UVM) (24). The patient survival data corresponding to certain cancer types (across 30 cancer types), including LIHC, such as ACC, BLCA, BRCA, CESC, CHOL, COAD, ESCA, HNSC, KICH, KIRC, KIRP, LUAD, LUSC, MESO, OV, PAAD, PCPG, PRAD, READ, SARC, SKCM, STAD, TGCT, THCA, THYM, UCEC, UCS and UVM, that were significantly associated with let-7c-5p expression were obtained using OncomiR online software (http://www.oncomir.org/) (25). This led to the identification of the miRNAs associated with tumor formation and patient survival.

Target genes of let-7c-5p and Kyoto Encyclopedia of Genes and Genomes (KEGG) pathway enrichment analysis. DIANA-TarBase v8 (http://www.microrna.gr/tarbase) is a reference database devoted to the indexing of experimentally supported miRNA targets (26). This database was used to screen the experimentally supported target genes of let-7c-5p (the filter value is default). Subsequently, the online biological tool KOBAS 3.0 (http://kobas.cbi.pku.edu.cn), which is a web server for gene/protein functional annotation (annotate module) and functional gene set enrichment, was used for KEGG (27) pathway enrichment analysis of the obtained target genes (28-30). The genes belonging to the PI3K-Akt and FoxO signaling pathways were selected for further analysis.

ONCOMINE analysis. Oncomine gene expression array datasets (http://www.oncomine.org; an online cancer microarray database) were used to display the gene summary view to analyze the mRNA differential expression levels of the let-7c-5p target genes that belonged to the PI3K-Akt and FoxO signaling pathways in 195 cancer vs. normal analyses (31). The thresholds were as follows: $\mathrm{P}<0.05$; fold-change $>1.5$; gene rank, ALL; data type, mRNA. The cancer specimen and normal control datasets were compared for each gene. The genes with different mRNA expression levels in the ONCOMINE analysis were selected for further analysis.

Kaplan-Meier plotter analysis. Kaplan-Meier plotter (www. kmplot.com) is an online database containing microarray gene expression data and survival information derived from Gene Expression Omnibus (32), TCGA (https://www.cancer. gov/tcga) and the Cancer Biomedical Informatics Grid (33), which contain mRNA expression data and survival information of 364 cases of clinical LIHC (34). These databases also contain miRNA expression data and survival information of 614 cases of clinical LIHC (35). To analyze overall survival (OS), all possible cut-off values between the lower and upper quartiles were computed and the best performing threshold was used as the cut-off, and the follow up threshold was restricted 60 months (5-year survival). The data were verified using a Kaplan Meier survival curve. In the present study, the associations between OS and let-7c-5p and its target genes obtained from ONCOMINE analysis were evaluated using 
the Kaplan-Meier plotter. Genes which were expressed at low levels and were associated with a high survival rate $(\mathrm{HR}>1)$ were selected for further analysis. In addition, the association between target gene expression data in different tumor grades and survival data was investigated.

LinkedOmics analysis. The LinkedOmics database (http://www.linkedomics.org/login.php) is a web-based platform for the analysis of 32 TCGA cancer-related datasets (36). In the present study, the correlation between let-7c-5p expression and the expression levels of its target genes in LIHC was analyzed using LinkedOmics. The target genes that exhibited a negative correlation with let-7c expression were selected for further analysis.

UALCAN analysis. UALCAN (http://ualcan.path.uab.edu) is an interactive web resource based on level 3 RNA sequencing methodology and the clinical data of 31 cancer types from TCGA. It is used to analyze the relative expression of genes in different tumor subgroups and of normal tumor samples with different tumor stages, tumor grades, ethnicity, weight or other clinicopathological characteristics (37). UALCAN was used to analyze the expression levels of the target genes that exhibited a negative correlation with let-7c-5p in different tumor grades of LIHC.

cBioPortal analysis. The cBioPortal for Cancer Genomics contained sequencing and pathological data of 30 different types of cancer $(38,39)$. The hepatocellular carcinoma dataset (derived from TCGA) containing 366 pathologically reported cases was selected for further analysis of the target genes obtained from the LinkedOmics dataset. In the setting of query parameters, mutations, assumed copy number changes from GISTIC and mRNA expression Z-scores (RNASeq V2 RSEM) were selected in 'Select Genomic Profiles', and the $\mathrm{z}$-score threshold was \pm 2.0 . The inputted target gene of let-7c-5p was analyzed using OncoPrint, Genetic Alteration, Mutual Exclusivity, Co-expression and the OS plotter.

Culture of MHCC-97H cell line. The LIHC MHCC-97H cell line (iCell Bioscience Inc., http://www.icellbioscience. com/cellDetail/568) was cultured in high-glucose DMEM (Gibco; Thermo Fisher Scientific, Inc.) supplemented with 10\% FBS (Gibco; Thermo Fisher Scientific, Inc.) and 1\% penicillin-streptomycin (Solarbio, Ltd.) at $37^{\circ} \mathrm{C}$ in a humidified incubator containing $5 \% \mathrm{CO}_{2}$.

Cell transfection. Prior to overexpression, MHCC-97H cells were plated in 6 -well culture plates, $\left(1 \times 10^{6} /\right.$ cell $)$. Once the MHCC-97H cells reached $80 \%$ confluence, they were transfected with hsa-let-7c-5p mimics (cat. no. 4464066, Assay ID: MC10436; Thermo Fisher Scientific, Inc., the overexpression group) or negative control miRNA mimic (cat. no. 4464058; Thermo Fisher Scientific, Inc. the overexpression control group) by using Lipofectamine ${ }^{\mathrm{TM}}$ RNAiMAX Transfection Reagent (Invitrogen; Thermo Fisher Scientific, Inc.), according to the manufacturer's protocol (https://assets.thermofisher. com/TFS-Assets/LSG/manuals/Lipofectamine_RNAiMAX_ Reag_protocol.pdf). After transfection, MHCC-97H cells were incubated at $37^{\circ} \mathrm{C}$ with $5 \% \mathrm{CO}_{2}$ for $48 \mathrm{~h}$. Each experiment was repeated 3 times.

Reverse transcription-quantitative $(R T-q) P C R$. Total RNA was extracted from each group using TRIzol ${ }^{\circledR}$ reagent (Invitrogen; Thermo Fisher Scientific, Inc.), according to the manufacturer's protocol. hsa-let-7c-5p was reverse transcribed and amplified using the TaqMan ${ }^{\text {TM }}$ MicroRNA assay (cat. no. 4427975, Assay ID: 000379; Thermo Fisher Scientific, Inc.) at the following conditions: $16^{\circ} \mathrm{C}$ for $30 \mathrm{~min}$, $42^{\circ} \mathrm{C}$ for $30 \mathrm{~min}$ and $85^{\circ} \mathrm{C}$ for $5 \mathrm{~min}$ (RT PCR), and $95^{\circ} \mathrm{C}$ for $10 \mathrm{~min}$ followed by 40 cycles of $95^{\circ} \mathrm{C}$ for $15 \mathrm{sec}$ and $60^{\circ} \mathrm{C}$ for $60 \mathrm{sec}$ (qPCR). To determine the expression levels of cyclin B2 (CCNB2), cyclin E2 (CCNE2), cyclin dependent kinase $4(\mathrm{CDK} 4)$, casein kinase $1 \varepsilon$ (CSNK1E), homer scaffold protein 1 (HOMER1), heat shock protein $90 \alpha$ family class A member 1 (HSP90AA1), neuroblastoma RAS viral oncogene homolog (NRAS), protein phosphatase 2 catalytic subunit $\alpha$ (PPP2CA), protein kinase AMP-activated catalytic subunit $\alpha 2$ (PRKAA2) and Rac family small GTPase 1 (RAC1), cDNAs were synthesized using the PrimeScript ${ }^{\mathrm{TM}}$ RT reagent kit (cat. no. RR037Q; Takara Bio, Inc.), and the primer sequences used for qPCR are listed in Table SI. qPCR was performed using a StepOnePlus ${ }^{\mathrm{TM}}$ system (Thermo Fisher Scientific, Inc.) and the TaqMan ${ }^{\circledR}$ Universal Master Mix II (cat. no. 4440043; Thermo Fisher Scientific, Inc.), with the following thermocycling conditions: $95^{\circ} \mathrm{C}$ for $10 \mathrm{~min}$ followed by 40 cycles of $95^{\circ} \mathrm{C}$ for $15 \mathrm{sec}$ and $60^{\circ} \mathrm{C}$ for $60 \mathrm{sec}$. U6 (cat. no. 4427975, Assay ID: 001973; Thermo Fisher Scientific, Inc.) and ACTB served as the internal controls for normalizing the relative expression levels of has-let-7c-5p and mRNA, respectively. Relative expression levels were calculated using the $2^{-\Delta \Delta \mathrm{Cq}}$ method (40).

Gene Ontology (GO) function analysis, KEGG pathway enrichment analysis and construction of protein-protein and gene-gene interaction networks. The functional analysis tool g:Profiler, gOST (rev fof4439; https://biit.cs.ut. ee/gprofiler/gost) was used to analyze GO functions $(41,42)$ and KEGG pathway enrichment $(43,44)$. GeneMANIA (http://genemania.org/) was used to construct gene-gene interaction networks $(45,46)$, and the Search Tool for the Retrieval of Interacting Genes/Proteins (STRING) database (version 11.0; https://string-db.org/) was used to construct the protein-protein interaction networks $(47,48)$.

Statistical analysis. OS was estimated by Kaplan-Meier analysis, and a log-rank test was performed to evaluate statistical significance. The co-expression and regression line of let-7c-5p and its target genes in LIHC tumors was determined by Pearson's correlation analysis. Co-expression among let-7c-5p target genes was determined by Spearman's correlation analysis. The association between the mRNA expression levels of the let-7c-5p target genes and the LIHC tumor grade was analyzed using Welch's test in a one-way heteroscedastic ANOVA with Games-Howell post hoc tests. For the expression difference of target genes between the let-7c-5p overexpression and control groups, the data are presented as the mean \pm standard deviation and analyzed by unpaired Student's t-test using SPSS v19 software (IBM 


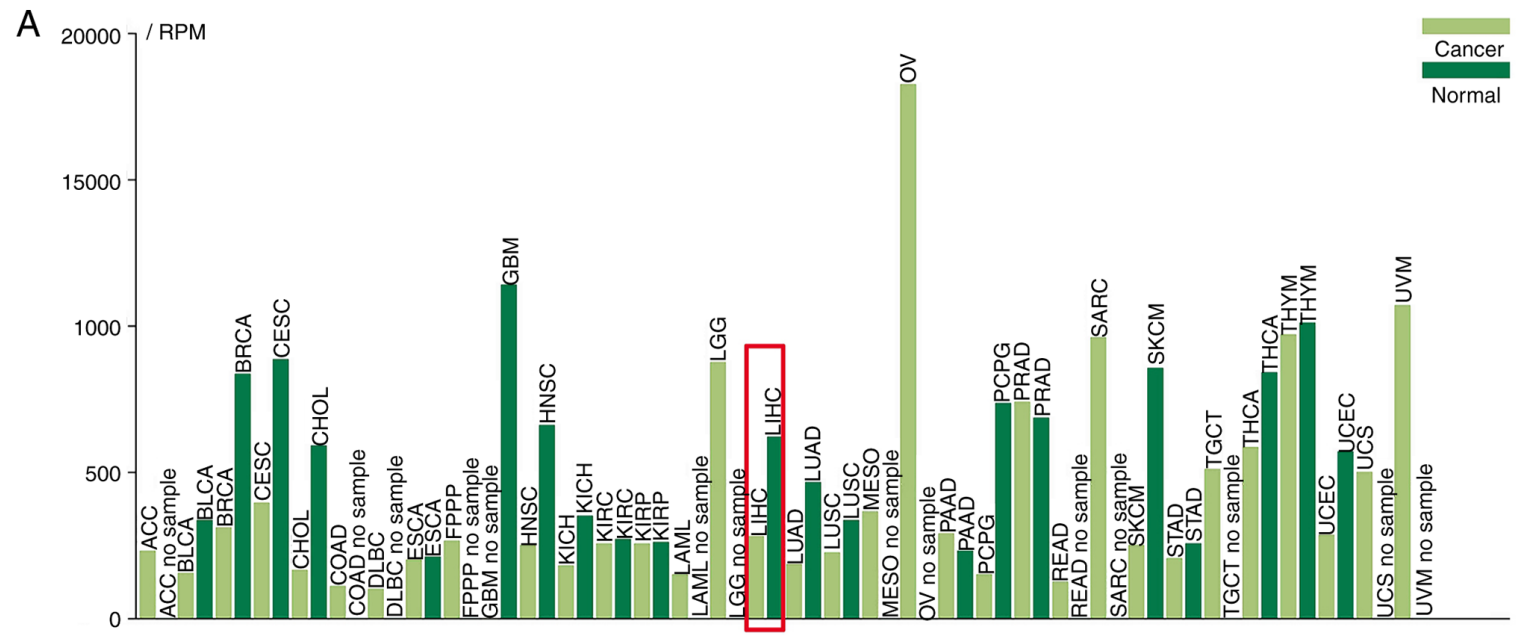

\begin{tabular}{|c|c|c|c|c|c|c|}
\hline B & $\begin{array}{c}\text { Cancer } \\
\text { miRNA Name }\end{array}$ & $\begin{array}{c}\text { Log Rank } \\
\text { Abbreviation }\end{array}$ & $\begin{array}{c}\text { Log Rank } \\
\text { FDalue }\end{array}$ & Z-score & Upregulated in: \\
\hline hsa-let-7c-5p & BLCA & $1.57 \mathrm{E}-04$ & $7.07 \mathrm{E}-02$ & 3.838 & Deceased \\
\hline hsa-let-7c-5p & HNSC & $1.38 \mathrm{E}-03$ & $7.14 \mathrm{E}-01$ & 3.208 & Living \\
\hline hsa-let-7c-5p & KICH & $4.89 \mathrm{E}-02$ & $6.12 \mathrm{E}-01$ & 2.032 & Living \\
\hline hsa-let-7c-5p & KIRC & $3.19 \mathrm{E}-02$ & $2.40 \mathrm{E}-01$ & 2.202 & Living \\
\hline hsa-let-7c-5p & LGG & $4.46 \mathrm{E}-02$ & $1.52 \mathrm{E}-02$ & 2.01 & Deceased \\
\hline hsa-let-7c-5p & LIHC & $3.16 \mathrm{E}-02$ & $9.86 \mathrm{E}-01$ & 2.195 & Living \\
\hline hsa-let-7c-5p & LUAD & $2.08 \mathrm{E}-02$ & $6.53 \mathrm{E}-01$ & 2.33 & Living \\
\hline hsa-let-7c-5p & PRAD & $4.43 \mathrm{E}-02$ & $6.24 \mathrm{E}-01$ & 2.049 & Living \\
\hline hsa-let-7c-5p & UVM & $1.35 \mathrm{E}-03$ & $1.05 \mathrm{E}-01$ & 3.32 & Living \\
\hline
\end{tabular}

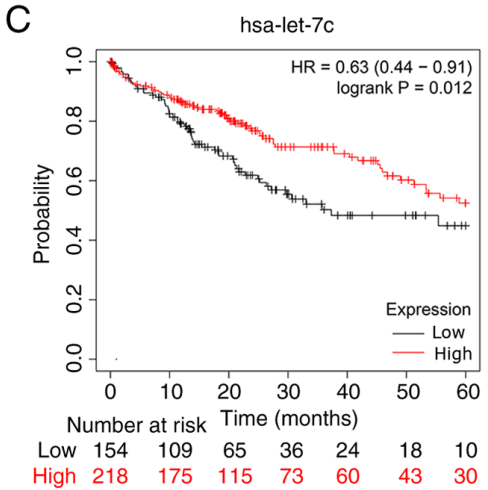

Figure 1. Clinical significance of let-7c-5p in LIHC as determined by TCGA analysis. (A) Expression profile of let-7c-5p as determined by TCGA obtained from Tumor-miRNA-Pathway. let-7c-5p expression was downregulated in LIHC tissues compared with in normal tissues. (B) Survival of patients with cancer and its association with let-7c-5p expression obtained from OncomiR. A total of 9 cancer types were identified. Patient survival was significantly associated with let-7c-5p expression, and the expression of let-7c-5p were upregulated in living cohorts in LIHC. (C) Kaplan-Meier curve obtained from Kaplan-Meier Plotter indicating the OS based on let-7c-5p levels in patients with LIHC. Low let-7c-5p expression was associated with poor OS of patients with LIHC. TCGA, The Cancer Genome Atlas; LIHC, liver hepatocellular carcinoma; miRNA, microRNA; OS, overall survival; FDR, false discovery rate; HR, hazard ratio; RPM, reads per million mapped reads.

Corp.). The data were representative of three independent experiments performed in triplicate. In all statistical analyses, $\mathrm{P}<0.05$ was considered to indicate a statistically significant difference.

\section{Results}

let-7c may act as a tumor suppressor gene in LIHC. The expression levels of let-7c-5p in different cancer types and their potential association with the survival rate were assessed using the online TCGA data analysis tool. The tumor-miRNA-pathway, OncomiR and the Kaplan-Meier plotter tools were used. let-7c-5p was differentially expressed in different cancer types compared with the corresponding expression noted in their control group, and its expression was decreased in LIHC (Fig. 1A). The survival rate of patients was significantly associated with let-7c-5p expression in 9 cancer types, while their performance was different. The expression of let-7c-5p were upregulated in living cohorts in LIHC (Fig. 1B). In addition, the Kaplan-Meier curve revealed that high let-7c-5p expression was associated with improved OS of patients with LIHC (Fig. 1C). Overall, these results demonstrated that let-7c-5p may act as a tumor suppressor gene in LIHC.
Target genes of let-7c are involved in the PI3K/Akt/FoxO signaling pathway in LIHC. Using TarBase v8.0 (Diana Tools), 2,051 target genes of let-7c were predicted. These target genes were subsequently used for KEGG pathway enrichment analysis using KOBAS 3.0. The data indicated that 58 target genes belonged to the 'PI3K-Akt signaling pathway', whereas 33 target genes belonged to the 'FoxO signaling pathway' (Table I). Among these genes, 15 target genes were associated with 'PI3K-Akt signaling pathway' and 'FoxO signaling pathway'. After excluding duplicate count genes, 76 let-7c-5p target genes associated with the PI3K/Akt/FoxO signaling pathway were identified.

Oncomine analysis was performed to detect the mRNA levels of these 76 let- $7 c-5 p$ target genes in different types of human cancer, including liver cancer. As presented in Fig. 2, the value in the cell presented the number of datasets with a statistically significant mRNA differential expression of let-7c-5p target genes. The results demonstrated that the differential expression of these let-7c-5p target genes was different between the liver tumor group and the normal group. For example, there were four upregulated and two downregulated analyses that met the thresholds for CCND2 and HSP90B1, respectively. However, for BCL2, there were four upregulated 
Table I. let-7c-5p target genes in the PI3K-Akt and FoxO signaling pathways.

\begin{tabular}{|c|c|c|c|}
\hline KEGG pathway & Number of genes & Corrected P-value & Genes \\
\hline PI3K-Akt signaling pathway & 58 & $5.81 \times 10^{-13}$ & $\begin{array}{l}\text { ATF2, ATF4, ATF6B, BCL2, BCL2L11, CCND1, } \\
\text { CCND2, CCNE2, CDK2, CDK4, CDK6, CDKN1A, } \\
\text { CDKN1B, CHRM1, CHUK, COL1A1, COL1A2, } \\
\text { COL4A1, COL4A2, CREB3L2, FGFR1, FN1, GNB1, } \\
\text { GNB2, GNG5, HSP90AA1, HSP90B1, IGF1R, IL6R, } \\
\text { INSR, ITGAV, JAK1, LAMC1, LPAR1, MAPK1, } \\
\text { MDM2, MET, MYC, NR4A1, NRAS, PKN2, PPP2CA, } \\
\text { PPP2CB, PPP2R1B, PPP2R5A, PPP2R5C, PRKAA1, } \\
\text { PRKAA2, PRLR, RAC1, RBL2, RPS6KB2, THBS1, } \\
\text { TLR4, TP53, TSC1, YWHAE, YWHAG }\end{array}$ \\
\hline FoxO signaling pathway & 33 & $3.46 \times 10^{-11}$ & $\begin{array}{l}\text { AGAP2, BCL2L11, CCNB2, CCND1, CCND2, CDK2, } \\
\text { CDKN1A, CDKN1B, CHUK, CSNK1E, FBXO32, } \\
\text { FOXO1, HOMER1, HOMER2, IGF1R, INSR, IRS2, } \\
\text { MAPK1, MAPK8, MDM2, NLK, NRAS, PLK2, } \\
\text { PRKAA1, PRKAA2, PRKAB2, PRKAG1, RBL2, SKP2, } \\
\text { MST1, TGFBR1, TGFBR2, TNFSF10 }\end{array}$ \\
\hline
\end{tabular}

KEGG, Kyoto Encyclopedia of Genes and Genomes.

and one downregulated analysis. The LIHC dataset of liver cancer was selected to assess the expression of target genes in detail, and the results demonstrated that 37 of the 76 genes were upregulated in LIHC, which opposed let-7c-5p expression (Table SII).

Using the Kaplan-Meier plotter analysis tool, the prognostic significance of the 37 genes obtained was assessed. It was identified that increased mRNA expression levels of CCNB2, CCNE2, CDK2, CDK4, CSNK1E, FBXO32, HOMER1, HSP90AA1, ITGAV, NRAS, PPP2CA, PRKAA2, RAC1 and SKP2 were associated with poor OS (Fig. 3), which suggested that the mRNA expression levels of the target genes of let-7c-5p may be useful for the prediction of the survival of patients with LIHC.

There were 367 overlapping samples with miRNASeq and RNASeq in the LinkedOmics database, and we used them to investigate the correlation of expression between let-7c and its target genes. The data were derived from the Kaplan-Meier plotter analysis of the LIHC samples. Additionally, Pearson's correlation analysis was used with $\mathrm{P}<0.01$ as the threshold value. The results indicated that the expression levels of 10 genes (CCNB2, CCNE2, CDK4, CSNK1E, HOMER1, HSP90AA1, NRAS, PPP2CA, PRKAA2 and RAC1) were negatively correlated with let-7c expression (Fig. 4), which suggested that let-7c-5p may regulate the expression of these genes in LIHC.

Expression levels of the let-7c-target genes are closely associated with tumor grade and LIHC prognosis. The present study aimed to identify potential candidate biomarkers for OS in patients with LIHC based on the mRNA expression levels of the target genes of let-7c-5p that were obtained from the LinkedOmics database. The comparisons between LIHC and liver tissues were performed using UALCAN. Higher mRNA expression levels of these genes (CCNB2, CCNE2,
CDK4, CSNK1E, HOMER1, HSP90AA1, NRAS, PPP2CA, PRKAA2 and RAC1) were noted in LIHC tissues compared with in normal tissues $(\mathrm{P}<0.05$; Fig. 5). Furthermore, the patients with more advanced stages of LIHC tended to exhibit higher expression levels of these target genes. Among them, the expression levels of the CCNB2 and CCNE2 were higher in grade 3 compared with in other grades $(\mathrm{P}<0.05)$. The expression levels of CSNK1E, NRAS, PPP2CA and RAC1 were higher in grade 3 compared with grade 1 . However, the expression levels of these target genes in tumor grade 4 did not exhibit this trend like in grade 3 , possibly due to the small sample size of grade 4 tumor samples.

The association between target gene expression data of different tumor grades and survival data was investigated (Fig. 6). High expression levels of CCNE2 were associated with poor OS in grade 1, 2 and 3 tumors. However, the expression levels of other genes were only associated with poor OS in one or two of the grades (data not shown). Overall, these results indicated that the mRNA expression levels of CCNE2 were associated with different tumor grades in patients with LIHC, and that their expression levels may be useful for the prediction of LIHC patient survival.

The performance of these 10 target genes (CCNB2, CCNE2, CDK4, CSNK1E, HOMER1, HSP90AA1, NRAS, PPP2CA, PRKAA2 and RAC1) was assessed in TCGA-LIHC, using cBioPortal. The results demonstrated that these 10 let-7c-5p target genes were altered in 201 out of 360 patients with LIHC (the total mutation rate was estimated to be $55.8 \%$; OncoPrint; Fig. 7A). CCNE2, PPP2CA and RAC1 were the three genes with the highest rate of sequence alterations and their mutation rates were estimated to be 23,18 and $12 \%$, respectively. KEGG pathway analysis demonstrated that 7 of the 10 target genes (CCNE2, CDK4, HSP90AA1, NRAS, PPP2CA, PRKAA2 and RAC1) belonged to the 'PI3K-Akt signaling pathway' and 5 target genes (CCNB2, CSNK1E, 

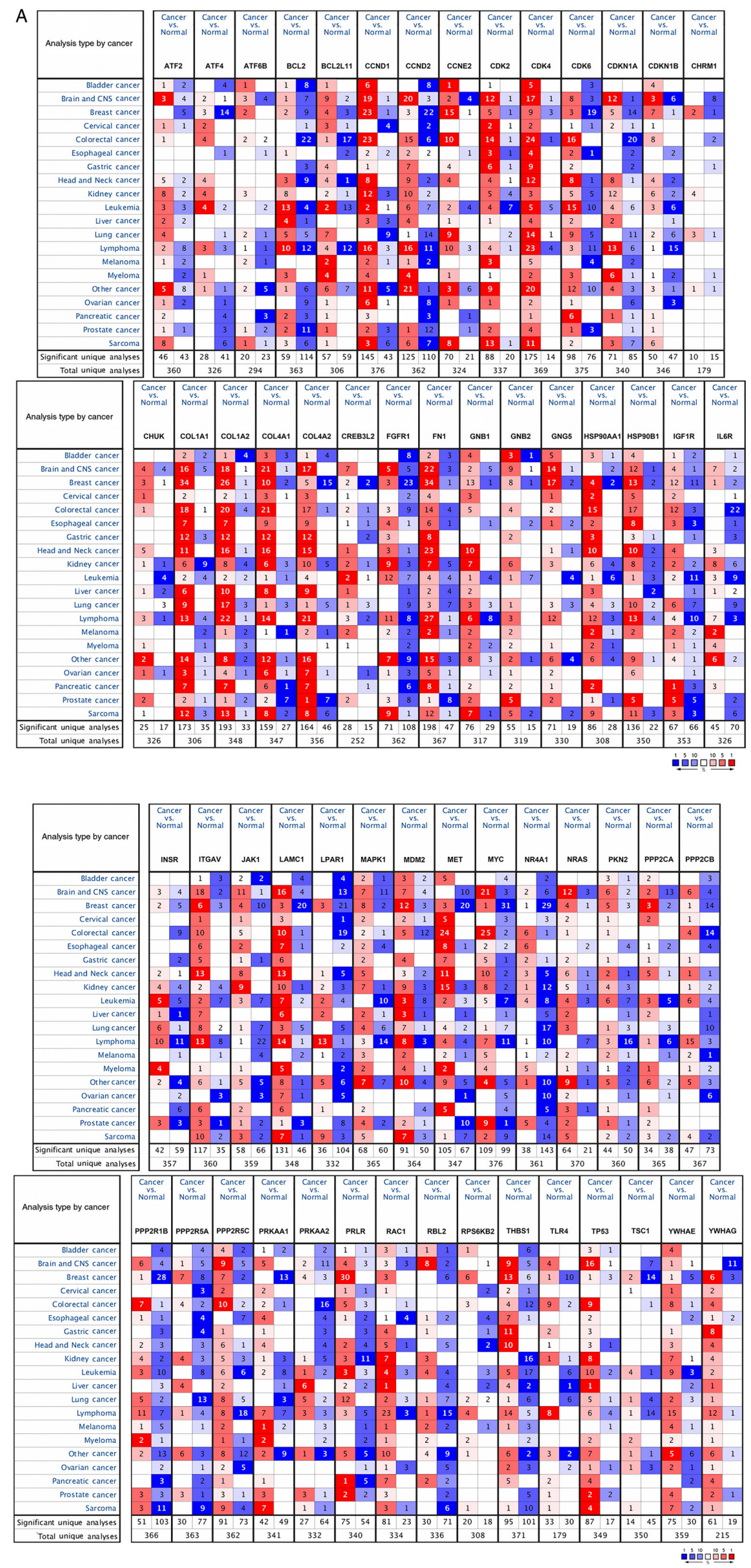

Figure 2. Continued. 


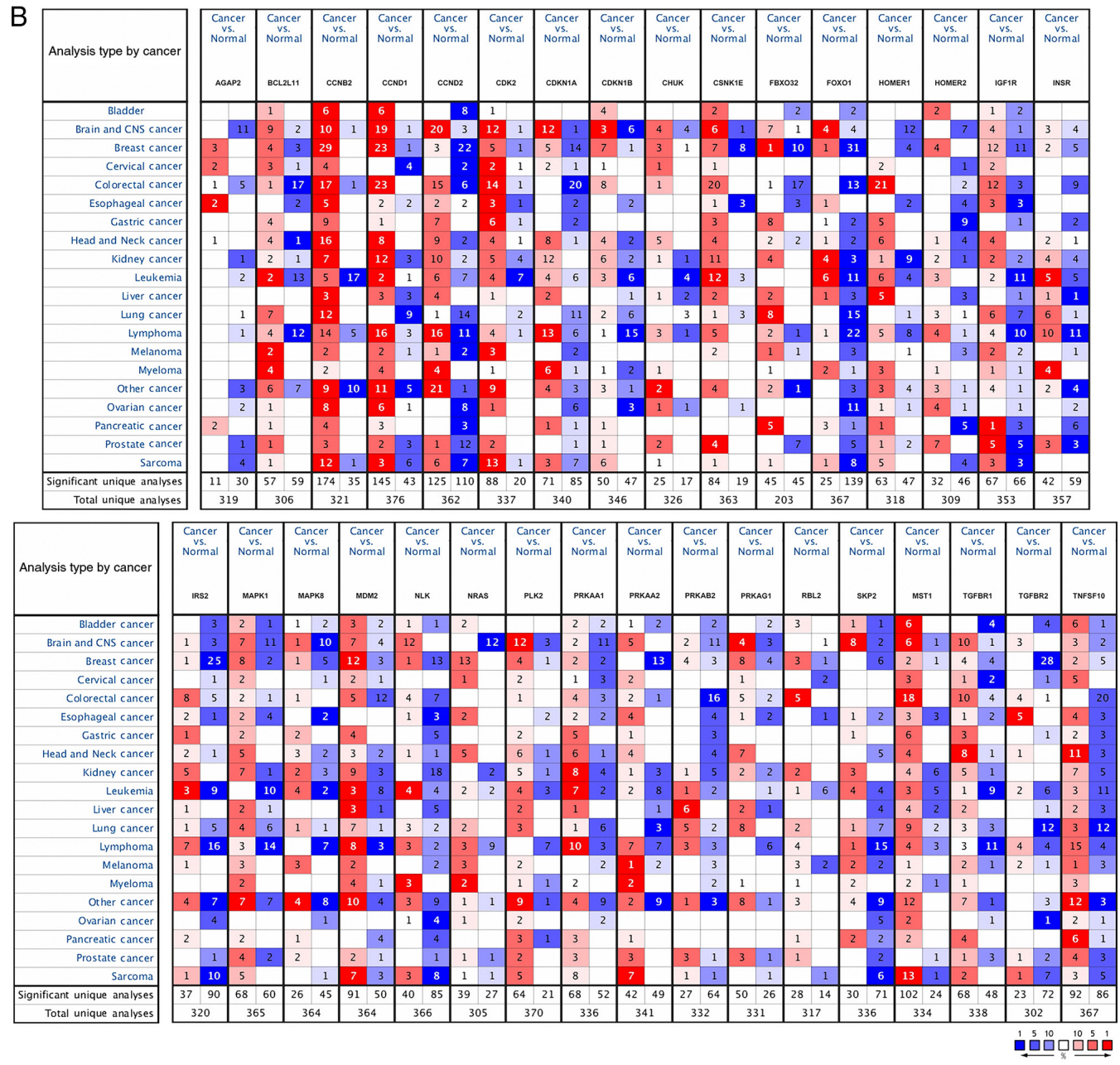

Figure 2. Disease summary for let-7c-5p target genes in different types of cancer obtained from Oncomine. The expression levels of the let-7c-5p target genes were assessed in 20 common types of cancer, and their levels were compared with normal individuals using the Oncomine database. The value in the cell presents the number of datasets with a statistically significant mRNA differential expression of let-7c-5p target genes, based on the cut-off value of $\mathrm{P}<0.05$ and fold change $>1.5$ in the Oncomine database. The cell color was determined by the best gene rank percentile for the analyses within the cell. Red represented high expression in tumors, blue represented low expression in tumors, and white represented no difference in tumor tissues and normal tissues. (A) Disease summary for let-7c-5p target genes that belong to the PI3K-Akt signaling pathway in different types of cancer. (B) Disease summary for let-7c-5p target genes that belong to the FoxO signaling pathway in different types of cancer.

HOMER1, NRAS and PRKAA2) to the 'FoxO signaling pathway'. Two of these genes, NRAS and PRKAA2, were shared by the two pathways (Fig. 7B). Further analysis of the alteration frequencies revealed that high mRNA levels were the most common alteration for CCNB2, CDK4, CSNK1E, HOMER1, HSP90AA1, NRAS, PPP2CA, PRKAA2 and RAC1. For CCNE2, the most common alteration was the amplification in LIHC tumors (Table SIII). Subsequently, the correlations of these 10 genes in LIHC tissues were investigated using Spearman's correlation (Fig. 7C). The results indicated a significant positive correlation among the majority of these genes, notably between CCNB2 and CCNE2 or CDK4. Spearman's correlation was estimated to be 0.63 and 0.62 , respectively, and the regression line is shown in Fig. 7C. The Spearman's correlation between CCNB2 and CSNK1E or NRAS, CCNE2 and CDK4 or NRAS, CDK4 and CSNK1E or RAC1 and HOMER1 and NRAS ranged between 0.3 and 0.6. Additional mutual exclusivity analysis indicated non-significant mutual exclusivity between two of these 10 genes (CCNB2, CCNE2, CDK4, CSNK1E, HOMER1, HSP90AA1,
NRAS, PPP2CA, PRKAA2 and RAC1), whereas the gene pairs of CCNB2 and CDK4, CSNK1E or NRAS, CDK4 and CSNK1E, HOMER1 or RAC1, HSP90AA1 and CCNE2 or NRAS, and NRAS and PRKAA2 or RAC1 exhibited significant $(\mathrm{P}<0.05)$ co-occurrence in LIHC (Fig. 7D). The selected samples in the TCGA-LIHC dataset were further divided into two groups based on whether the 10 target genes were altered. One group was the altered group: Samples with at least one alteration in the 10 let-7c-5p target genes in the selected profiles, and the other group was the unaltered group: Samples without any alterations in the 10 let-7c-5p target genes in the selected profiles. Further analysis using the Kaplan-Meier plotter and a log-rank test indicated that the alterations of these 10 genes were associated with worse OS in patients with LIHC (Fig. 7E). These suggested that alterations of these 10 genes could have an impact on the prognosis of patients with LIHC.

A total of nine genes may be the target genes of let-7c-5p, and their main function is the regulation of the cell cycle. Following let-7c-5p overexpression in MHCC-97H, it was 

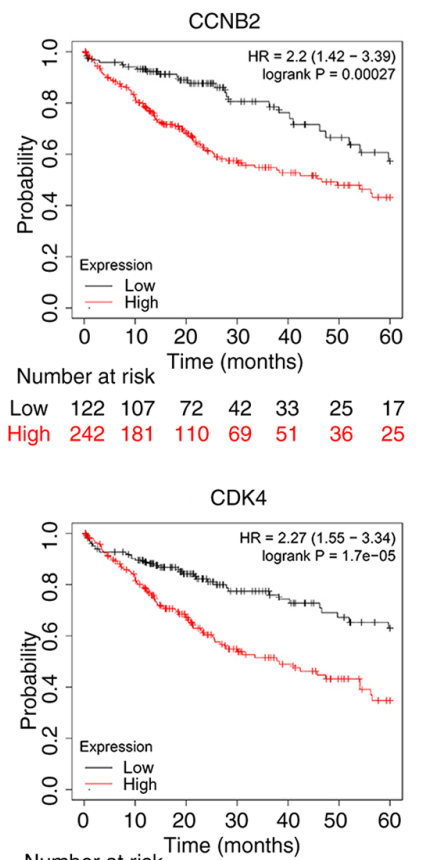

Number at risk Time (months)

$\begin{array}{llllllll}\text { Low } & 167 & 143 & 93 & 59 & 47 & 36 & 28\end{array}$ $\begin{array}{llllllll}\text { High } & 197 & 145 & 89 & 52 & 37 & 25 & 14\end{array}$

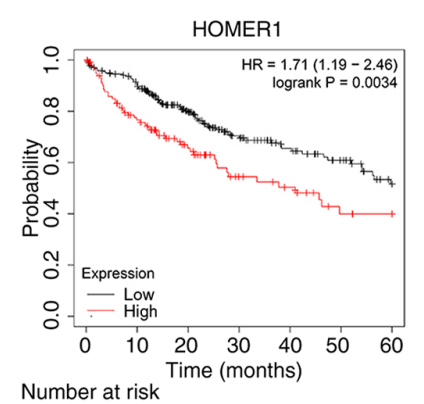

Low $243 \begin{array}{llllll}208 & 132 & 83 & 61 & 47 & 30\end{array}$

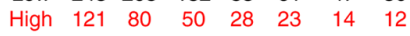
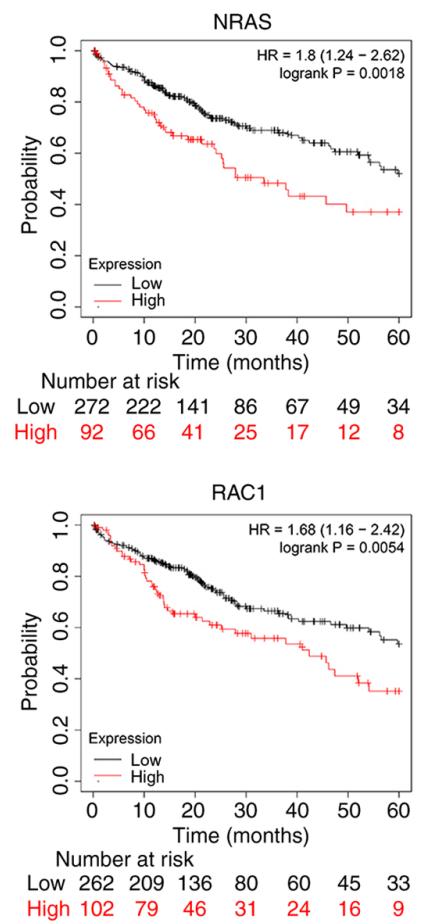

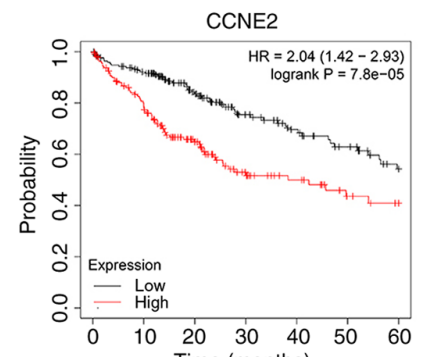

Number at risk Time (months)

Low $\begin{array}{lllllll}199 & 168 & 112 & 71 & 55 & 43 & 29\end{array}$

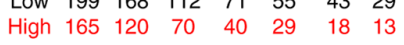
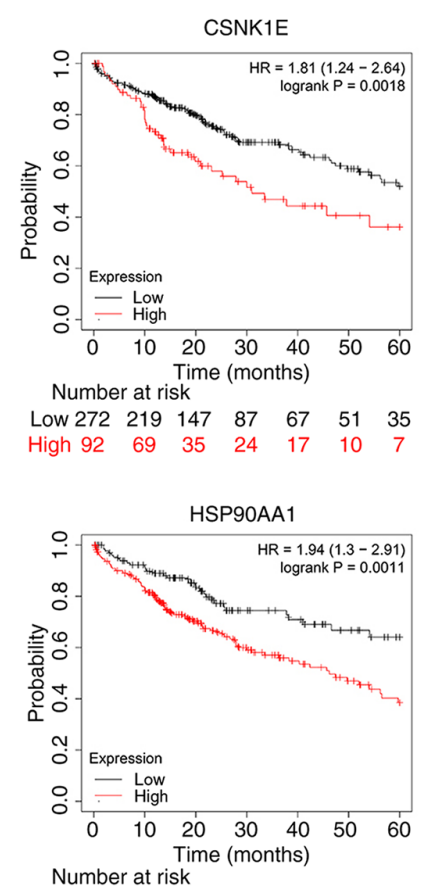

Number at risk

Low $\begin{array}{lllllll}137 & 113 & 80 & 46 & 39 & 28 & 20\end{array}$ High $\begin{array}{lllllll}227 & 175 & 102 & 65 & 45 & 33 & 22\end{array}$
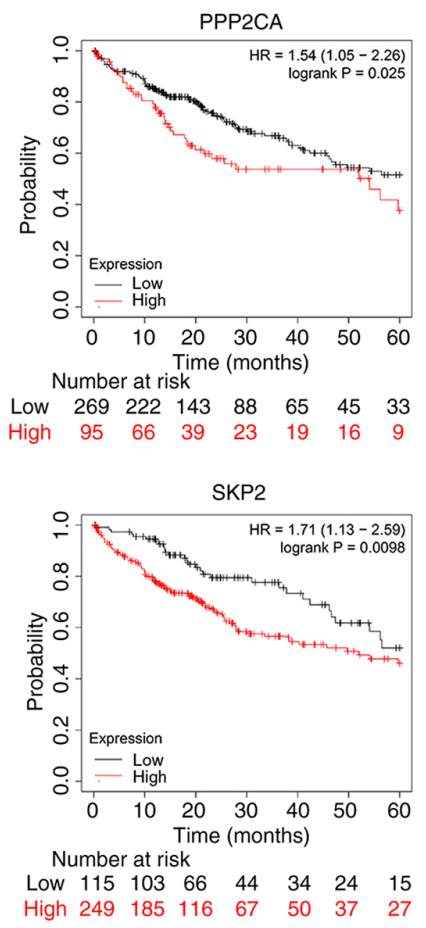
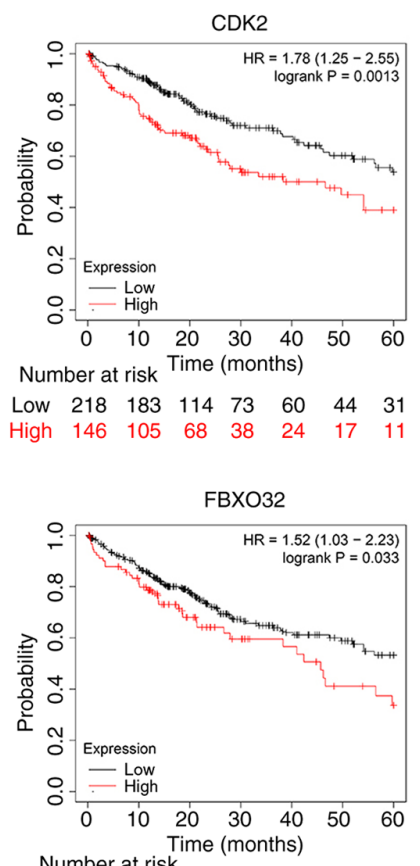

Number at risk

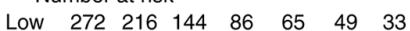
$\begin{array}{lccccccc}\text { High } & 92 & 72 & 38 & 25 & 19 & 12 & 9\end{array}$
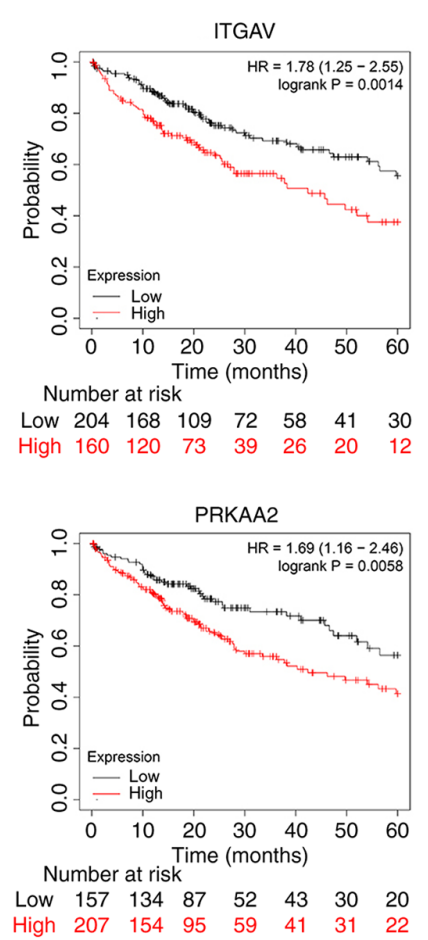

Figure 3. Prognostic value of let-7c-5p-target gene mRNA levels in liver hepatocellular carcinoma by using Kaplan-Meier Plotter. Increased mRNA expression levels of CCNB2, CCNE2, CDK2, CDK4, CSNK1E, FBXO32, HOMER1, HSP90AA1, ITGAV, NRAS, PPP2CA, PRKAA2, RAC1 and SKP2 were associated with poor overall survival $(\mathrm{P}<0.05)$. HR, hazard ratio. 

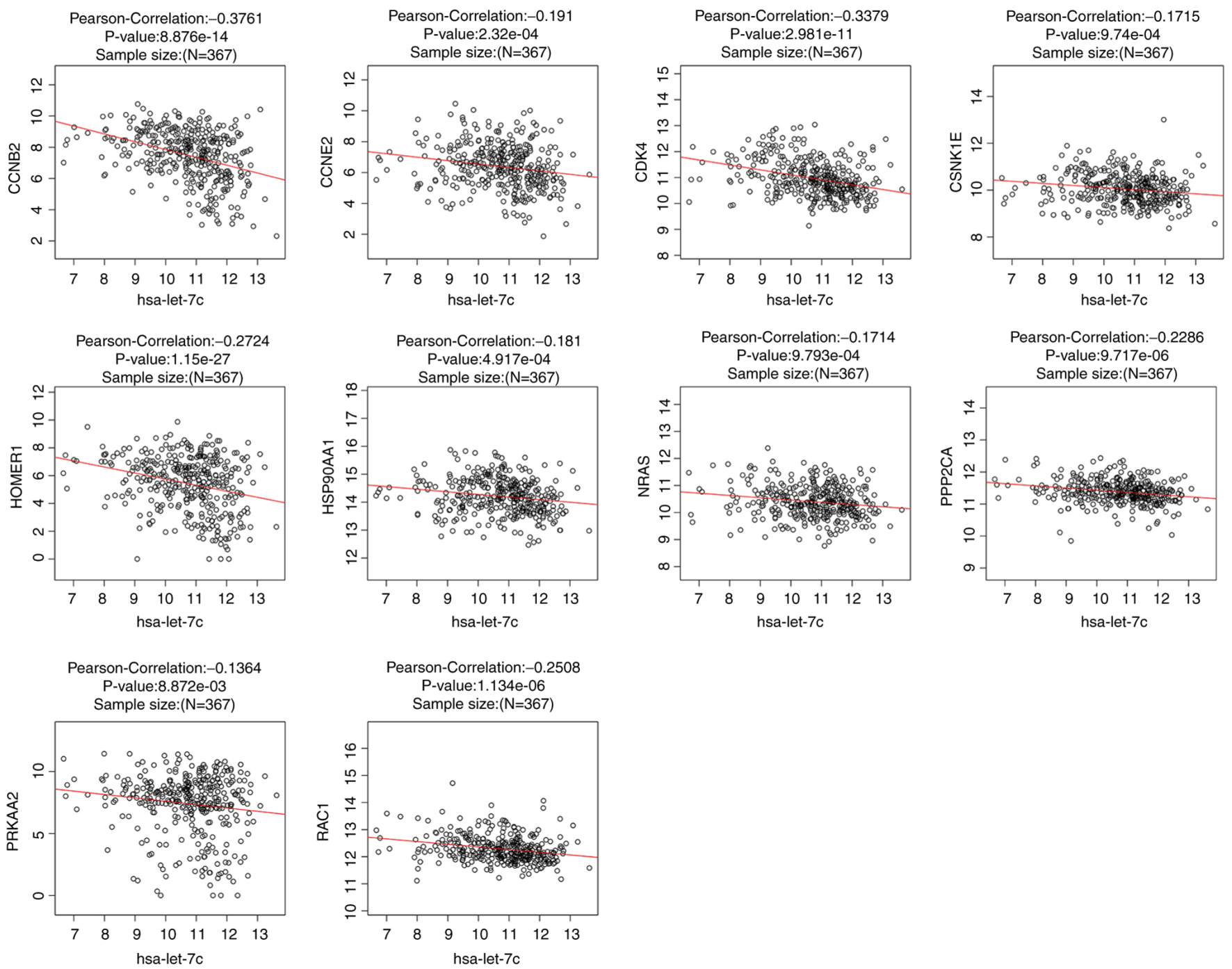

Figure 4. Correlation between let-7c-5p expression and the expression levels of its target genes in liver hepatocellular carcinoma obtained from LinkedOmics. mRNA expression levels of CCNB2, CCNE2, CDK4, CSNK1E, HOMER1, HSP90AA1, NRAS, PPP2CA, PRKAA2 and RAC1 were negatively correlated with let-7c $(\mathrm{P}<0.01)$.

revealed that there was no significant difference in the expression levels of CSNK1E compared with the control group. However, the expression levels of CCNB2, CCNE2, CDK4, HOMER1, HSP90AA1, NRAS, PPP2CA, PRKAA2 and RAC1 were downregulated to different degrees, which demonstrated that these genes may be target genes of let-7c (Fig. 8). Subsequently, g:Profiler was used to analyze GO function and KEGG pathway enrichment for these nine genes, while GO analysis was used to divide gene functions into biological process (BP), molecular function (MF) and cellular component (CC) categories. According to the number of genes clustered to the corresponding terms (Intersection size) and the correlation with LIHC disease, the results of the present study demonstrated that the genes were mainly clustered into the following: The MF terms included 'phosphotransferase activity, alcohol group as acceptor' and 'cyclin-dependent protein serine/threonine kinase regulator activity'. The BP terms included 'protein phosphorylation' and 'cell cycle process', and the CC terms included 'cytosol'. Among the genes, seven belonged to the 'PI3K-Akt signaling pathway' and four belonged to the 'FoxO signaling pathway' (Fig. 9).
GeneMANIA software was used to analyze associations in terms of co-expression, physical interactions, shared protein domains, predicted, pathway and co-localization among these nine target genes of let-7c-5p (20 additional genes were involved in the network), and to highlight the functions associated with the majority of these genes (Fig. 10A). The results indicated the following processes: ' $\mathrm{G}_{2} / \mathrm{M}$ transition of mitotic cell cycle', ' $\mathrm{G}_{1} / \mathrm{S}$ transition of mitotic cell cycle', 'cell cycle $\mathrm{G}_{2} / \mathrm{M}$ phase transition', 'Ras protein signal transduction' and 'cell cycle checkpoint'. Subsequently, STRING was used to analyze these genes in terms of their protein expression levels. The results indicated complex interactions among these genes (Fig. 10B). Overall, these results indicated complex links among the target genes, whose main function was the regulation of the cell cycle. Changes in the expression levels of these genes may have an important effect on the cell cycle.

\section{Discussion}

Accumulating evidence has indicated that miRNAs are abnormally expressed in LIHC tumors and promote or inhibit the 

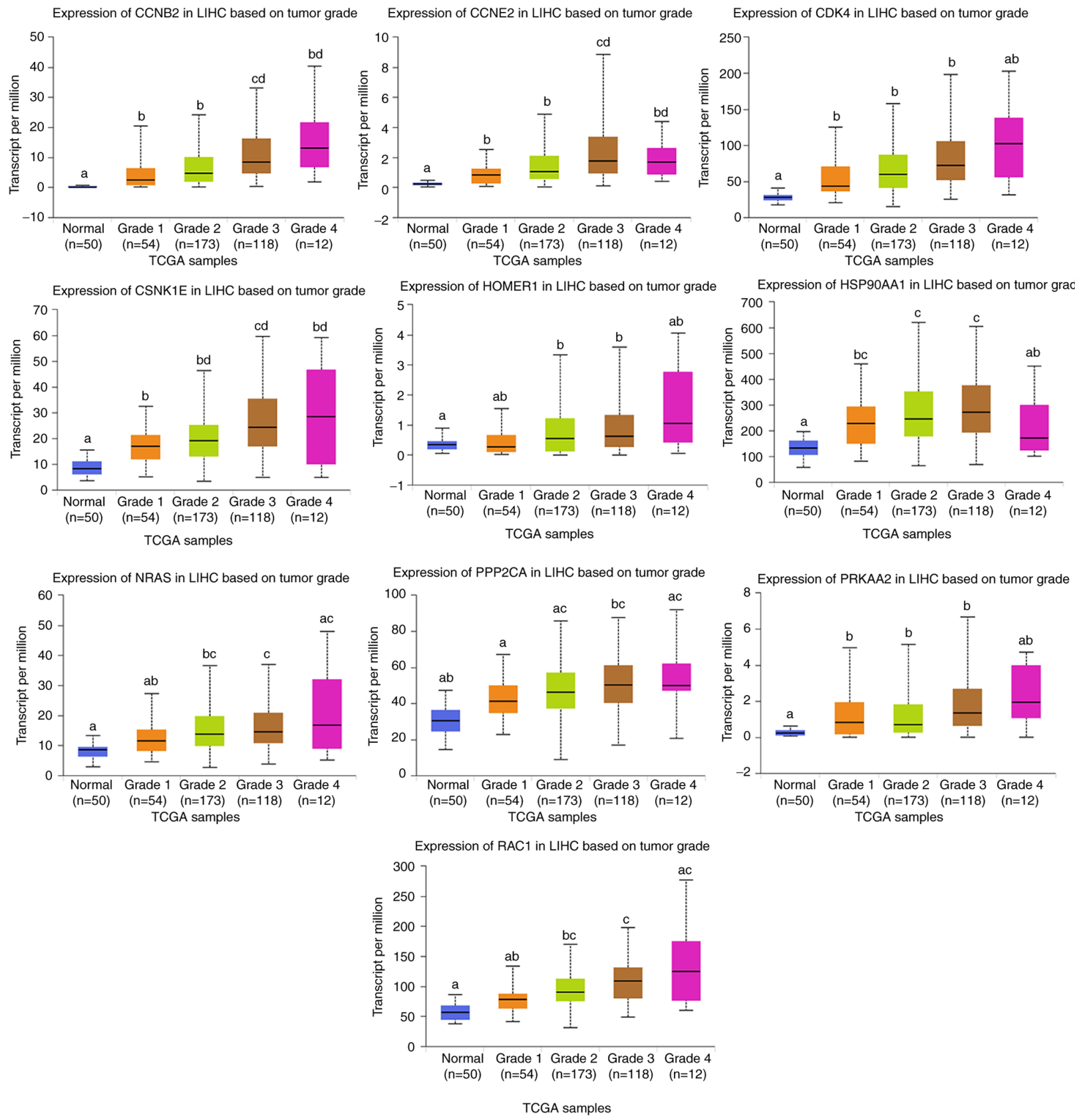

Figure 5. Association between the mRNA expression levels of the let-7c-5p target genes and LIHC tumor grade as determined using TCGA data obtained from UALCAN. Groups labeled with different letters were significantly different from each other $(\mathrm{P}<0.05)$, whereas groups labeled with the same letter were not. LIHC, liver hepatocellular carcinoma; TCGA, The Cancer Genome Atlas.
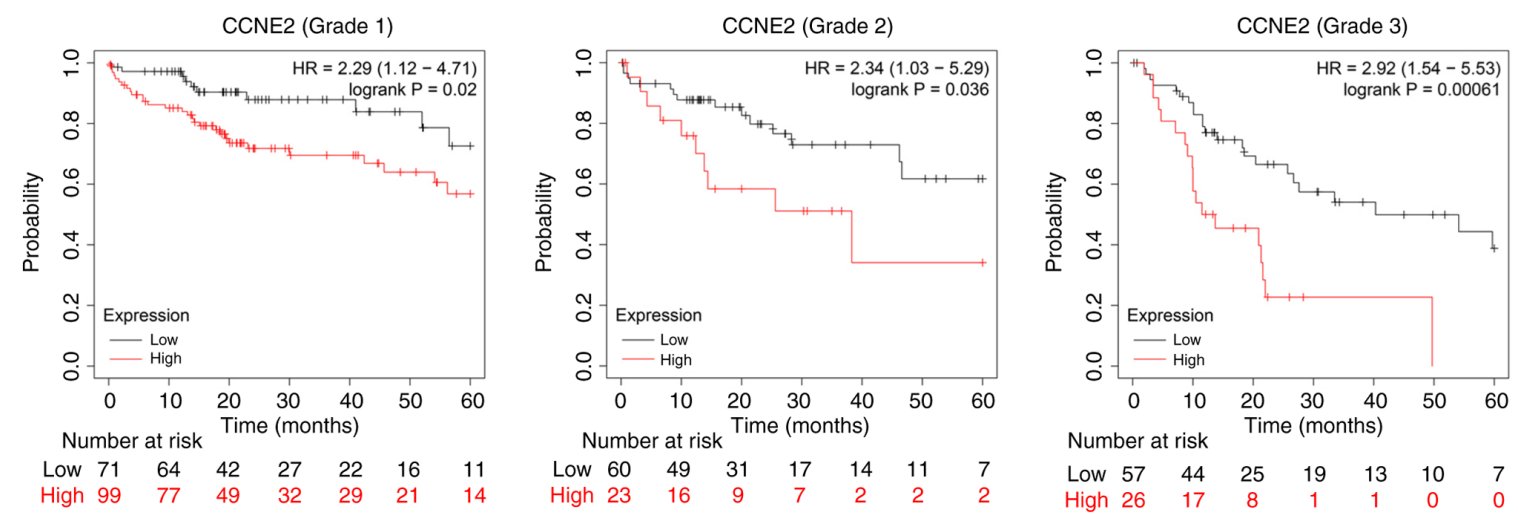

Figure 6. Association between the expression levels of CCNE2 in grade 1, 2 or 3 tumors and patient survival obtained from Kaplan-Meier Plotter. High expression levels of CCNE2 were associated with significantly poorer overall survival in grade 1, 2 and 3 tumors $(\mathrm{P}<0.05)$. HR, hazard ratio. 
A

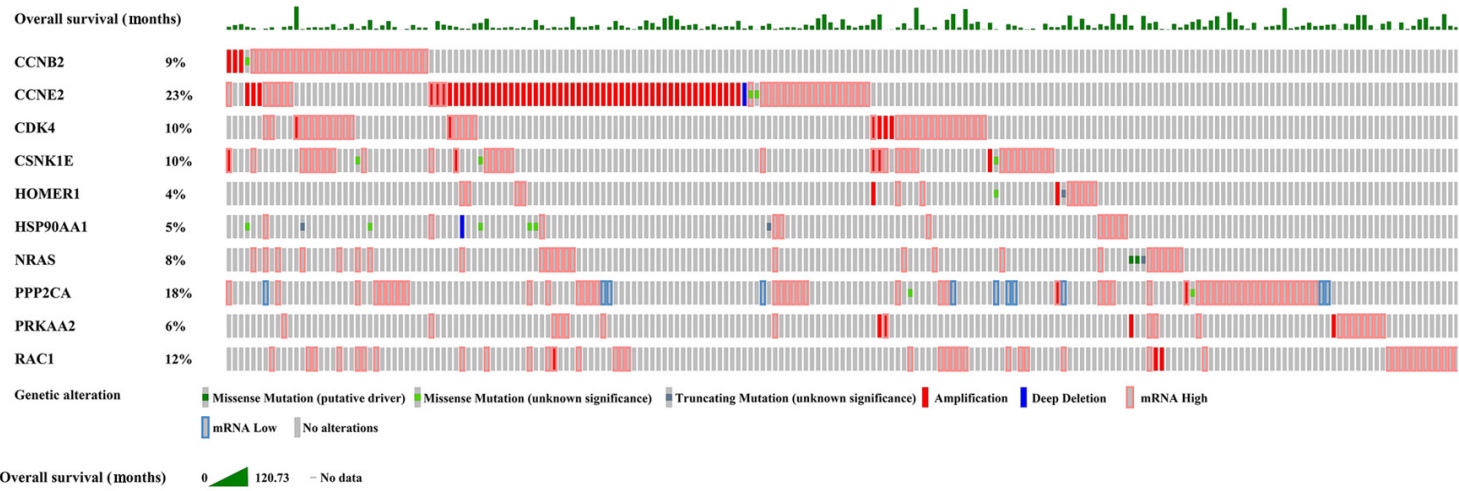

B
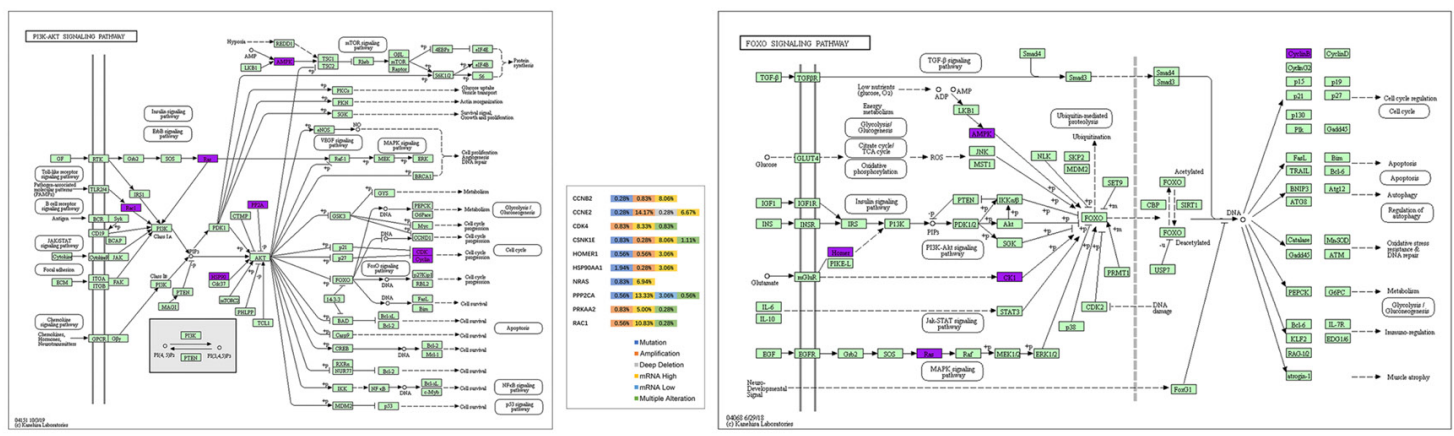

$\mathrm{C}$
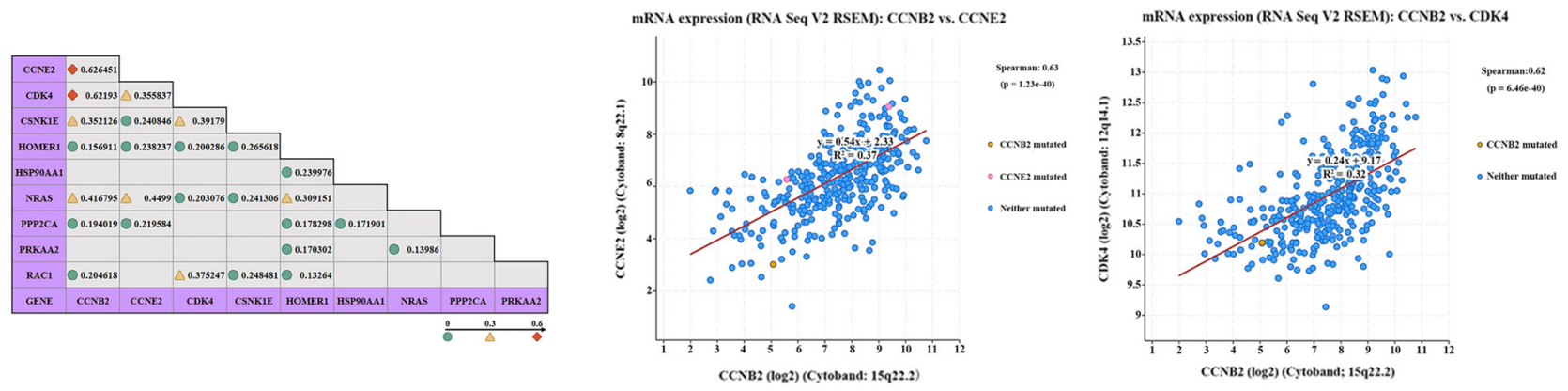

$\mathrm{D}$

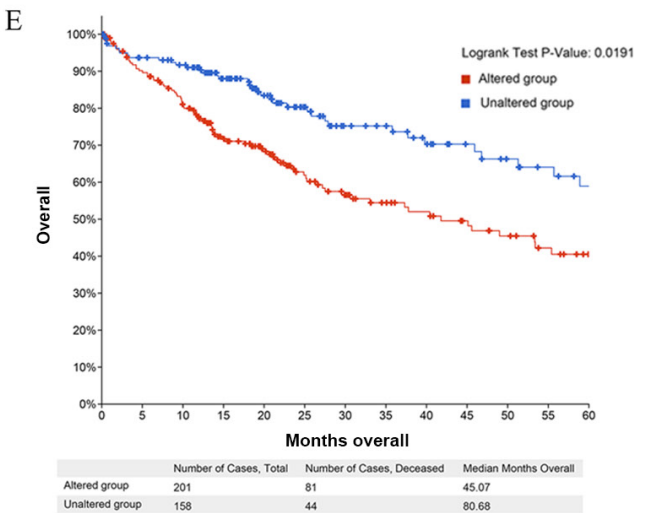

Figure 7. cBioPortal data visualization and analysis. (A) Oncoprint of genetic alterations and OS observed for CCNB2, CCNE2, CDK4, CSNK1E, HOMER1, HSP90AA1, NRAS, PPP2CA, PRKAA2 and RAC1 in LIHC tumors. (B) Detailed alteration frequencies of CCNB2, CCNE2, CDK4, CSNK1E, HOMER1, HSP90AA1, NRAS, PPP2CA, PRKAA2 and RAC1 in LIHC and their position in the PI3K-Akt and FoxO signaling pathways. KEGG pathway analysis demonstrated that 7 of the 10 target genes (CCNE2, CDK4, HSP90AA1, NRAS, PPP2CA, PRKAA2 and RAC1) belonged to the 'PI3K-Akt signaling pathway' and 5 target genes (CCNB2, CSNK1E, HOMER1, NRAS and PRKAA2) to the 'FoxO signaling pathway'. The genes in purple represent the let-7c-5p target genes. The alteration frequencies revealed that high mRNA levels were the most common alteration for CCNB2, CDK4, CSNK1E, HOMER1, HSP90AA1, NRAS, PPP2CA, PRKAA2 and RAC1. For CCNE2, the most common alteration was the amplification in LIHC tumors. (C) Co-expression of CCNB2, CCNE2, CDK4, CSNK1E, HOMER1, HSP90AA1, NRAS, PPP2CA, PRKAA2 and RAC1 in LIHC tumors. The regression line of CCNB2 with CCNE2 and CCNB2 with CDK4 was determined by Spearman's correlation analysis (Spearman's Correlation $>0.6 ; \mathrm{P}<0.05$ ). (D) Mutual exclusivity of CCNB2, CCNE2, CDK4, CSNK1E, HOMER1, HSP90AA1, NRAS, PPP2CA, PRKAA2 and RAC1 in LIHC tumors. The values in the table represent the $\log 2$ Odds Ratio. $\log 2$ ratio $>0$ indicates a tendency towards co-occurrence $(\mathrm{P}<0.05)$. (E) Kaplan-Meier plots comparing OS in cases with/without CCNB2, CCNE2, CDK4, CSNK1E, HOMER1, HSP90AA1, NRAS, PPP2CA, PRKAA2 and RAC1 gene alterations in LIHC tumors. The samples were divided into two groups according to whether the 10 target genes were altered. One group was the altered group: Samples in which at least one of the 10 target genes was altered (201 in total), and one group was the unaltered group: Samples with no alteration in all 10 target genes (158 in total). The analysis using the Kaplan-Meier plotter and a log-rank test indicated that the alterations of these 10 genes were associated with worse OS in patients with LIHC. LIHC, liver hepatocellular carcinoma; OS, overall survival. 


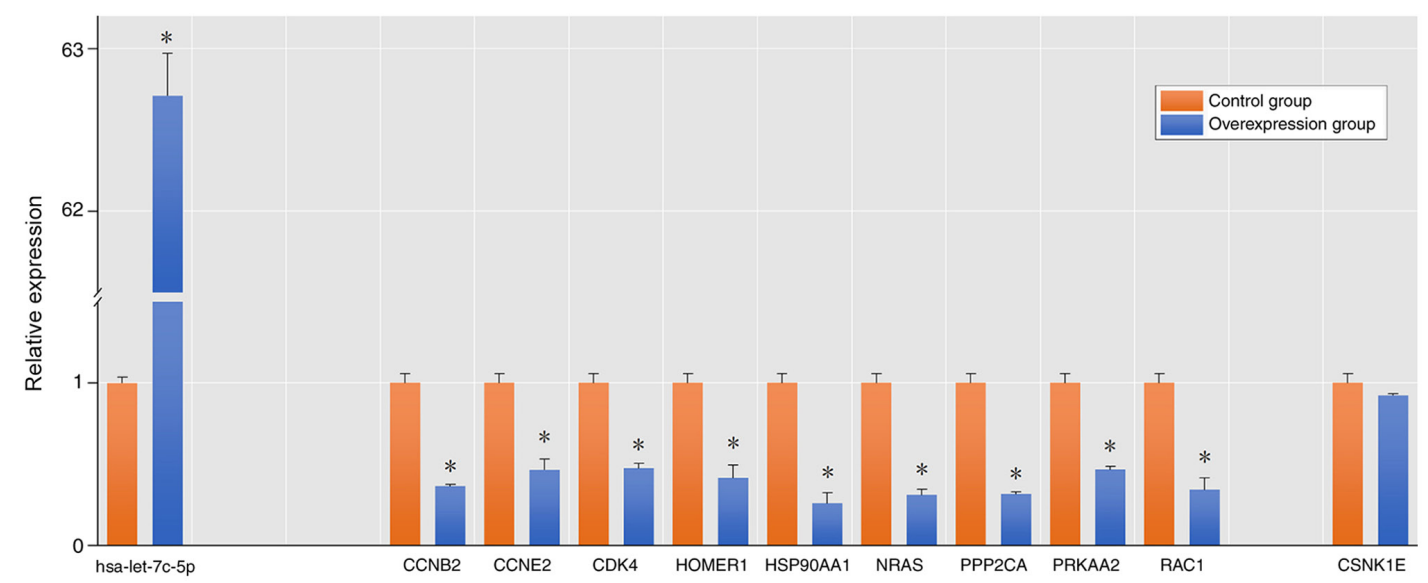

Figure 8 Overexpression of hsa-let-7c-5p in MHCC-97H cells induced the downregulation of CCNB2, CCNE2, CDK4, HOMER1, HSP90AA1, NRAS, PPP2CA, PRKAA2 and RAC1 expression. However, no significant difference was observed between the expression levels of CSNK1E in the overexpression group and those in the control group. ${ }^{*} \mathrm{P}<0.05$ vs. control group.

development and progression of LIHC by affecting cell cycle and proliferation, metastasis and invasion, drug resistance and inhibition of apoptosis (49-51). It has been demonstrated that the levels of let-7c are lower in LIHC tissues than in the corresponding normal adjacent tissues (52). In addition, a previous study indicated that let-7c inhibits cell proliferation and induces cell cycle arrest in LIHC tissues (15). In the present study, let-7c expression was decreased in LIHC tissues and this was associated with poor OS of LIHC as determined by the online TCGA data analysis tools Tumor-miRNA-Pathway, OncomiR and Kaplan-Meier plotter. Jilek et al (53) identified that let-7c bioengineered preparations could inhibit LIHC development. Overall, these results indicated that let-7c may be an effective therapeutic target for LIHC. However, since each miRNA can regulate thousands of target genes, not all target genes of let-7c expressed in LIHC and are only regulated by it. Therefore, the exact role of let-7c in the development and progression of LIHC remains to be elucidated. The PI3K/Akt signaling pathway has been recognized as one of the most frequently activated signal transduction pathways in cancer (17), whereas the FoxO signaling pathway has been mainly investigated for the potential targeting of cancer therapeutic molecules and proteins (54). The FoxO signaling pathway is closely associated with the PI3K/Akt signaling pathway (55). The analysis of the association between the target genes of let-7c and the PI3K/Akt and FoxO signaling pathways could further aid the understanding of the role of let-7c in LIHC and provide a basis for the future application of let-7c in LIHC therapy. In the present study, the expression levels and prognostic values of let-7c and its target genes in patients with LIHC were analyzed using multiple online tools.

Initially, 2,051 target genes of let-7c were identified by DIANA-TarBase v8 analysis and 76 target genes were identified that belonged to the PI3K-Akt and FoxO signaling pathways using KOBAS 3.0 and KEGG pathway enrichment analysis. Oncomine was used to analyze mRNA transcription levels, and Kaplan-Meier plotter was used to evaluate OS. A total of 37 target genes of let-7c-5p were upregulated in LIHC and 14 genes among them were associated with poor OS. Finally, 10 genes were negatively associated with let-7c expression according to LinkedOmics. Furthermore, in vitro experiments revealed that nine genes among them were downregulated after let-7c-5p overexpression in MHCC-97H cells, which suggested that let-7c-5p regulated the expression of these genes in LIHC (Fig. 11). Additionally, analysis using Kaplan-Meier Plotter highlighted that the high expression levels of CCNE2 were associated with poor OS in grade 1,2 and 3 tumors. The results suggested that these nine genes, including CCNE2, may be promising candidate biomarkers for disease and poor prognosis in LIHC. KEGG analysis demonstrated that let-7c did not directly regulate the expression of PI3K, Akt and FoxO in LIHC, but mainly that of its target genes that were upstream molecules of the PI3K-Akt and the FoxO signaling pathways, which can directly regulate PI3K, Akt and FoxO, such as NRAS, RAC1, HSP90AA1, PPP2CA, and PRKAA2, or their downstream molecules, such as CCNB2, CCNE2 and CDK4.

It is well-known that the cell cycle serves an important role in cancer. GO analysis indicated that six (CCNB2, CCNE2, CDK4, HSP90AA1, PPP2CA and PRKAA2) of these nine target genes of let-7c were members of the 'cell cycle process' BP term. Among them, CCNB2 is an important member of the cyclin protein family and is an important cell cycle regulator associated with $\mathrm{G}_{2} / \mathrm{M}$ detection sites (56). High expression levels of CCNB2 are negatively associated with the OS of patients with LIHC (57). The principal function of CCNE2 is to be a regulator to facilitate the cell cycle progression from $G_{0} / G_{1}$ to $S$ phase $(58,59)$. Upregulation of CCNE2 is associated with cancer progression and mortality in several types of cancer (60). CDK4 serves a key role in the proliferation of mammalian cells and can promote the cell entry to the $\mathrm{S}$ phase of the cell cycle (61). HSP90AA1 is a chaperone that is highly conserved among eukaryotes (62). Functional HSP90 is required for the stability of Akt, which is a key gene of the PI3K-Akt signaling pathway and serves a crucial regulatory role in differentiation, cell cycle, transcription, translation, metabolism and apoptosis (63). PPP2CA serves critical roles in several cellular processes by dephosphorylating critical cellular molecules, such as Akt, P53, c-Myc and $\beta$-catenin (64). Notably, downregulation of PRKAA2 has been reported in breast tumors and other types of cancer $(65,66)$, which suggests that it possesses tumor suppressor functions in vivo. However, the data indicated that PRKAA2 expression 


\begin{tabular}{|c|c|c|c|c|c|c|c|c|c|c|c|c|c|c|c|}
\hline \multicolumn{2}{|l|}{ GO:MF } & \multicolumn{5}{|l|}{ stats } & \multirow{2}{*}{ 孛 } & & \multirow{2}{*}{$\mathcal{O}$} & \multirow{2}{*}{\multicolumn{2}{|c|}{ 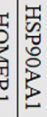 }} & \multirow{2}{*}{ 壳 } & \multirow{2}{*}{ 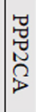 } & & \multirow{2}{*}{$\begin{array}{l}2 \\
2\end{array}$} \\
\hline Term name & Term id & Padj & $-\log 10($ Padj $)$ & Term size & Query size & Intersection size & & & & & & & & & \\
\hline cyclin-dependent protein serine/threonine kinase regulator activity & GO:0016538 & $2.19 \mathrm{E}-04$ & & 47 & 9 & 3 & $\checkmark$ & $ه$ & $\checkmark$ & & & & & & \\
\hline protein kinase regulator activity & GO:0019887 & $1.29 \mathrm{E}-02$ & & 182 & 9 & 3 & $\nabla$ & $\checkmark$ & $\checkmark$ & & & & & & \\
\hline kinase regulator activity & GO:0019207 & $2.03 \mathrm{E}-02$ & & 212 & 9 & 3 & $\checkmark$ & $\checkmark$ & $\checkmark$ & & & & & & \\
\hline protein kinase activity & GO:0004672 & $2.32 \mathrm{E}-02$ & 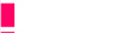 & 615 & 9 & 4 & 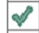 & $\checkmark$ & $\checkmark$ & & & & & $\checkmark$ & \\
\hline protein kinase binding & GO:0019901 & $2.98 \mathrm{E}-02$ & 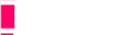 & 656 & 9 & 4 & $\checkmark$ & $\nabla$ & & & $\checkmark$ & & & & $\checkmark$ \\
\hline tau protein binding & GO:0048156 & $3.46 \mathrm{E}-02$ & & 45 & 9 & 2 & & & & & $\nabla$ & จ & $\nabla$ & & \\
\hline phosphotransferase activity, alcohol group as acceptor & GO:0016773 & $4.27 \mathrm{E}-02$ & & 720 & 9 & 4 & $\nabla$ & $\nabla$ & $\checkmark$ & & & & & $\checkmark$ & \\
\hline kinase binding & GO:0019900 & $4.82 \mathrm{E}-02$ & [ & 743 & 9 & 4 & $\nabla$ & $\nabla$ & & & 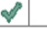 & & & & $\checkmark$ \\
\hline
\end{tabular}

\begin{tabular}{|c|c|c|c|c|c|c|c|c|c|c|c|c|c|c|c|}
\hline \multicolumn{2}{|l|}{ GO:BP } & \multicolumn{5}{|l|}{ stats } & \multirow{2}{*}{ 总 } & \multirow{2}{*}{ 疍 } & \multirow{2}{*}{$\underset{Ð}{\Theta}$} & & \multirow{2}{*}{ 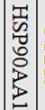 } & & & \multirow{2}{*}{$\begin{array}{l}\text { 帘 } \\
\text { 员 } \\
⿱\end{array}$} & \multirow{2}{*}{ 恣 } \\
\hline Term name & Term id & Padj & $-\log 10($ Padj $)$ & Term size & Query size & Intersection size & & & & & & & & & \\
\hline protein phosphorylation & GO:0006468 & $2.87 \mathrm{E}-04$ & & 2038 & 9 & 8 & $\sqrt{7}$ & $\checkmark$ & $\checkmark$ & & $\checkmark$ & ? & 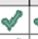 & $\checkmark s$ & $\checkmark$ \\
\hline phosphorylation & GO:0016310 & $1.40 \mathrm{E}-03$ & & 2492 & 9 & 8 & $\checkmark$ & $\checkmark$ & $\checkmark$ & & $\checkmark$ & $\checkmark$ & $\checkmark$ & $\checkmark$ & $\checkmark$ \\
\hline regulation of phosphorylation & GO:0042325 & $2.36 \mathrm{E}-03$ & & 1670 & 9 & 7 & $\checkmark$ & $\checkmark$ & $\checkmark$ & & $\checkmark$ & & $\checkmark$ & $\checkmark$ & $\checkmark$ \\
\hline regulation of phosphate metabolic process & GO:0019220 & $5.11 \mathrm{E}-03$ & & 1871 & 9 & 7 & 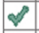 & $\checkmark$ & $\nabla$ & & $\checkmark$ & & $\checkmark$ & $\checkmark$ & $\checkmark$ \\
\hline regulation of phosphorus metabolic process & GO:0051174 & $5.15 \mathrm{E}-03$ & & 1873 & 9 & 7 & $\checkmark$ & $\checkmark$ & $\checkmark$ & & $\nabla$ & & $\$$ & $\checkmark$ & $\checkmark$ \\
\hline regulation of protein modification process & GO:0031399 & $6.12 \mathrm{E}-03$ & & 1921 & 9 & 7 & $\nabla$ & 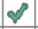 & $\checkmark$ & & $\$$ & & 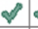 & $\checkmark$ & $\checkmark$ \\
\hline phosphate-containing compound metabolic process & GO:0006796 & $1.53 \mathrm{E}-02$ & & 3380 & 9 & 8 & $\nabla$ & $\checkmark$ & $\checkmark$ & & $\checkmark$ & $\checkmark$ & $\nabla$ & $\checkmark$ & $\checkmark$ \\
\hline phosphorus metabolic process & GO:0006793 & $1.63 \mathrm{E}-02$ & & 3406 & 9 & 8 & $\nabla$ & $\checkmark$ & $\checkmark$ & & $\checkmark$ & $\checkmark$ & $\checkmark$ & $\nabla$ & $\checkmark$ \\
\hline regulation of cyclin-dependent protein serine/threonine kinase activity & GO:0000079 & $1.72 \mathrm{E}-02$ & & 99 & 9 & 3 & $\checkmark$ & $\checkmark$ & $\checkmark$ & & & & & & \\
\hline regulation of cyclin-dependent protein kinase activity & GO: 1904029 & $1.94 \mathrm{E}-02$ & & 103 & 9 & 3 & $\checkmark$ & $\checkmark$ & $\checkmark$ & & & & & & \\
\hline cell cycle process & GO:0022402 & $2.00 \mathrm{E}-02$ & & 1403 & 9 & 6 & $\nabla$ & $\checkmark$ & $\checkmark$ & & $\checkmark$ & & $\nabla$ & $\nabla$ & \\
\hline regulation of protein kinase activity & GO:0045859 & $2.59 \mathrm{E}-02$ & & 810 & 9 & 5 & $\nabla$ & $\checkmark$ & 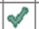 & & $\checkmark$ & & 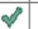 & & \\
\hline response to metal ion & GO:0010038 & $2.66 \mathrm{E}-02$ & & 370 & 9 & 4 & & & $\checkmark$ & $\nabla$ & & & 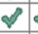 & $\checkmark$ & \\
\hline regulation of protein phosphorylation & GO:0001932 & $2.97 \mathrm{E}-02$ & & 1502 & 9 & 6 & $\checkmark$ & $\checkmark$ & $\checkmark$ & & $\checkmark$ & & $\checkmark$ & & 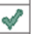 \\
\hline mitotic cell cycle process & GO:1903047 & 4.13E-02 & & 892 & 9 & 5 & $\checkmark$ & $\$$ & $\checkmark$ & & $\nabla$ & & $\Phi$ & & \\
\hline regulation of kinase activity & GO:0043549 & $4.80 \mathrm{E}-02$ & & 920 & 9 & 5 & $\nabla$ & & $\checkmark$ & & $\checkmark$ & & $\nabla$ & & \\
\hline
\end{tabular}

\begin{tabular}{|c|c|c|c|c|c|c|c|c|c|c|c|c|c|c|c|}
\hline \multicolumn{2}{|l|}{ GO:CC } & \multicolumn{5}{|l|}{ stats } & \multirow{2}{*}{ 罣 } & \multirow{2}{*}{ 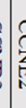 } & \multirow{2}{*}{$\underset{\triangle}{\Theta}$} & & \multirow{2}{*}{ 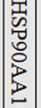 } & & & & \multirow{2}{*}{ 营 } \\
\hline Term name & Term id & Padj & $-\log 10\left(\right.$ Padj $_{16}$ & Term size & Query size & Intersection size & & & & & & & & & \\
\hline cyclin-dependent protein kinase holoenzyme complex & GO:0000307 & $8.95 \mathrm{E}-05$ & & 42 & 9 & 3 & $\nabla$ & $\$$ & $\checkmark$ & & & & & & \\
\hline serine/threonine protein kinase complex & GO: 1902554 & $7.89 \mathrm{E}-04$ & & 86 & 9 & 3 & $\checkmark$ & $\checkmark$ & $\checkmark$ & & & & & & \\
\hline protein kinase complex & GO:1902911 & $1.32 \mathrm{E}-03$ & & 102 & 9 & 3 & $\checkmark$ & $\checkmark$ & $\checkmark$ & & & & & & \\
\hline dendrite & GO:0030425 & 1.37E-02 & & 624 & 9 & 4 & & & & $\checkmark$ & $\checkmark$ & & & $\nabla$ & $\checkmark$ \\
\hline dendritic tree & GO:0097447 & $1.39 \mathrm{E}-02$ & & 626 & 9 & 4 & & & & $\nabla$ & $\infty$ & & & 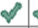 & $\checkmark$ \\
\hline cytosol & GO:0005829 & $2.24 \mathrm{E}-02$ & & 5161 & 9 & 8 & $\nabla$ & $\checkmark$ & $\downarrow$ & $\$$ & 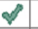 & & $\$ 8$ & 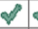 & $\checkmark$ \\
\hline transferase complex, transferring phosphorus-containing groups & GO:0061695 & $2.34 \mathrm{E}-02$ & & 268 & 9 & 3 & $\checkmark$ & $\nabla$ & $\checkmark$ & & & & & & \\
\hline somatodendritic compartment & GO:0036477 & $4.82 \mathrm{E}-02$ & 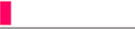 & 865 & 9 & 4 & & & & & $\nabla$ & & & $\nabla$ & $\checkmark$ \\
\hline
\end{tabular}

\begin{tabular}{|c|c|c|c|c|c|c|c|c|c|c|c|c|c|c|c|}
\hline \multicolumn{2}{|l|}{ KEGG } & \multicolumn{5}{|l|}{ stats } & \multirow{2}{*}{ 灾 } & \multirow{2}{*}{ 畺 } & & \multirow{2}{*}{ 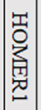 } & \multirow{2}{*}{ 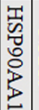 } & \multirow{2}{*}{ 兑 } & & & \multirow{2}{*}{ 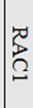 } \\
\hline Term name & Term id & Padj & $-\log 10\left(\mathrm{Padj}_{16}\right.$ & Term size & Query size & Intersection size & & & & & & & & & \\
\hline PI3K-Akt signaling pathway & KEGG:04151 & $6.66 \mathrm{E}-07$ & & 353 & 9 & 7 & & 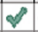 & $\checkmark$ & & $\checkmark$ & $\checkmark$ & $\nabla$ & $\checkmark$ & $\checkmark$ \\
\hline FoxO signaling pathway & KEGG:04068 & $4.51 \mathrm{E}-04$ & & 129 & 9 & 4 & $\checkmark$ & & & $\nabla$ & & $\checkmark$ & & $\nabla$ & \\
\hline Cellular senescence & KEGG:04218 & $9.59 \mathrm{E}-04$ & & 156 & 9 & 4 & $\nabla$ & $\checkmark$ & $\checkmark$ & & & $\checkmark$ & & & \\
\hline Tight junction & KEGG:04530 & $1.06 \mathrm{E}-03$ & & 160 & 9 & 4 & & & $\checkmark$ & & & & $\nabla$ & $\nabla$ & $\checkmark$ \\
\hline Viral carcinogenesis & KEGG:05203 & $2.65 \mathrm{E}-03$ & & 202 & 9 & 4 & & 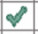 & $\checkmark$ & & & $\checkmark$ & & & $\checkmark$ \\
\hline p53 signaling pathway & KEGG:04115 & 3.37E-03 & & 73 & 9 & 3 & $\checkmark$ & $\infty$ & $\checkmark$ & & & & & & \\
\hline Human T-cell leukemia virus 1 infection & KEGG:05166 & $3.45 \mathrm{E}-03$ & & 216 & 9 & 4 & $\checkmark$ & $\checkmark$ & $\checkmark$ & & & $\checkmark$ & & & \\
\hline Pathways in cancer & KEGG:05200 & $7.58 \mathrm{E}-03$ & & 529 & 9 & 5 & & $\nabla$ & $\checkmark$ & & $\checkmark$ & $\checkmark$ & & & $\checkmark$ \\
\hline Prostate cancer & KEGG:05215 & $7.88 \mathrm{E}-03$ & & 97 & 9 & 3 & & $\checkmark$ & & & $\checkmark$ & $\checkmark$ & & & \\
\hline AGE-RAGE signaling pathway in diabetic complications & KEGG:04933 & $8.62 \mathrm{E}-03$ & & 100 & 9 & 3 & & & 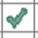 & & & $\checkmark$ & & & $\checkmark$ \\
\hline Sphingolipid signaling pathway & KEGG:04071 & $1.44 \mathrm{E}-02$ & & 119 & 9 & 3 & & & & & & $\checkmark$ & $\checkmark$ & & $\checkmark$ \\
\hline Cell cycle & KEGG:04110 & $1.63 \mathrm{E}-02$ & & 124 & 9 & 3 & $\nabla$ & $\checkmark$ & $\checkmark$ & & & & & & \\
\hline Oocyte meiosis & KEGG:04114 & $1.71 \mathrm{E}-02$ & & 126 & 9 & 3 & $\checkmark$ & $\checkmark$ & & & & & $\nabla$ & & \\
\hline Human papillomavirus infection & KEGG:05165 & $1.79 \mathrm{E}-02$ & & 330 & 9 & 4 & & 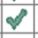 & $\checkmark$ & & & $\checkmark$ & $\nabla$ & & \\
\hline Autophagy - animal & KEGG:04140 & $2.14 \mathrm{E}-02$ & & 136 & 9 & 3 & & & & & & $\checkmark$ & 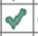 & $\checkmark$ & \\
\hline Fluid shear stress and atherosclerosis & KEGG:05418 & $2.19 \mathrm{E}-02$ & & 137 & 9 & 3 & & & & & $\checkmark$ & & & $\nabla$ & $\checkmark$ \\
\hline Hepatitis C & KEGG:05160 & $3.14 \mathrm{E}-02$ & & 155 & 9 & 3 & & & $\checkmark$ & & & $\checkmark$ & $\nabla$ & & \\
\hline Bladder cancer & KEGG:05219 & $5.00 \mathrm{E}-02$ & & 41 & 9 & 2 & & & $\checkmark$ & & & $\checkmark$ & & & \\
\hline
\end{tabular}

Figure 9. GO function and KEGG pathway enrichment for CCNB2, CCNE2, CDK4, HOMER1, HSP90AA1, NRAS, PPP2CA, PRKAA2 and RAC1 in liver hepatocellular carcinoma tumors. The results were summarized in the following three main categories: BP, MF and CC. GO, Gene Ontology; KEGG, Kyoto Encyclopedia of Genes and Genomes; BP, biological process; MF, molecular function; CC, cellular component.

was upregulated in LIHC. The role of this gene in LIHC is not clear and requires further study. Therefore, it was deduced that the majority of these target genes of let-7c-can affect the cell cycle of LIHC cells via the PI3K-Akt and FoxO signaling pathways and that their high expression may aggravate cell cycle disorders. The data suggest that the high expression levels of these target genes, notably CCNE2, are associated with poor prognosis of LIHC. HOMER1 has been reported to interact with $\mathrm{Ca}^{+2}$ signaling proteins, such as stromal interaction molecule 1 and ORAI calcium release-activated calcium modulator 1 (67). Alterations in either the expression or the activity of these proteins have been associated with the onset 
A

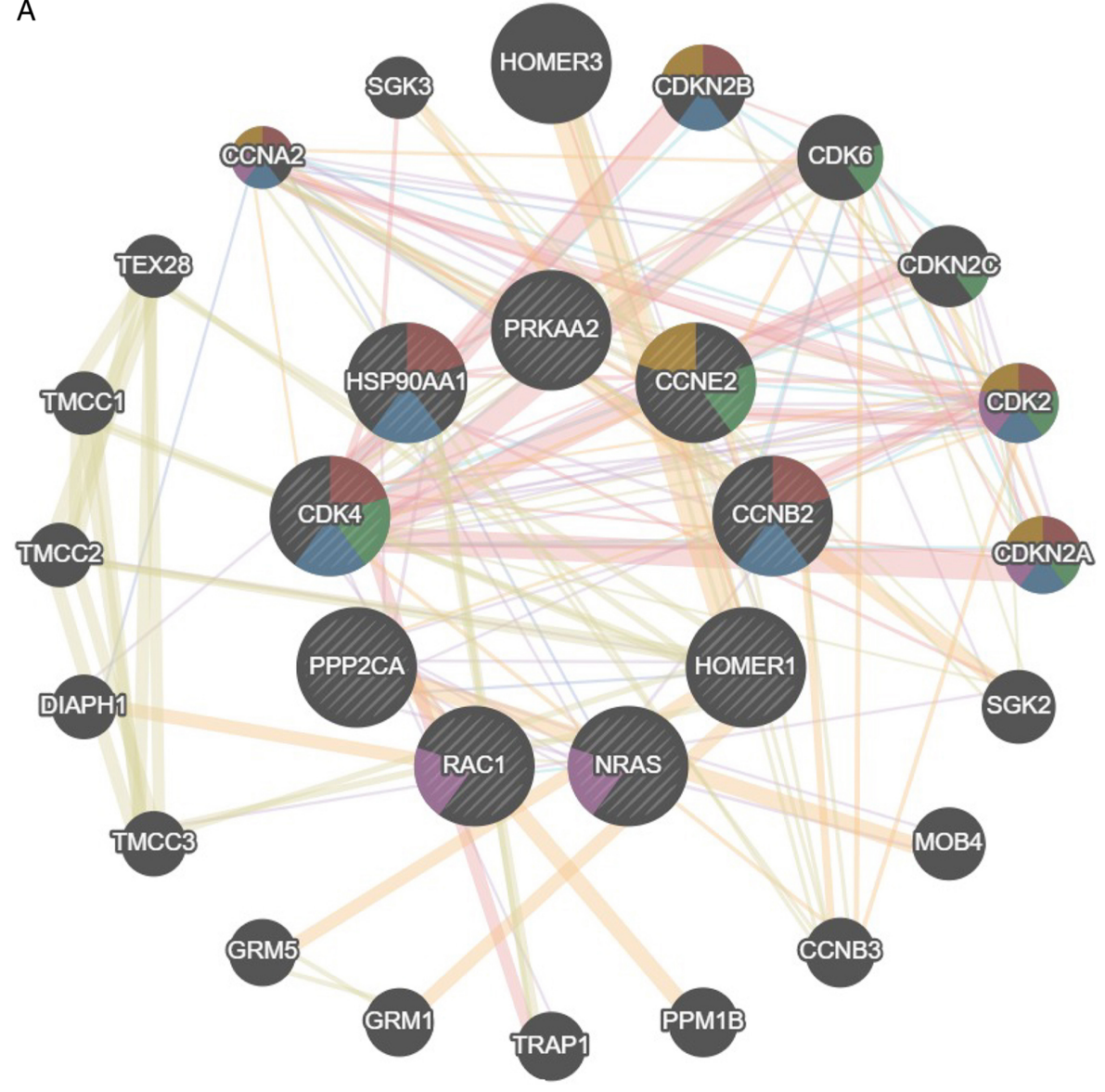

\begin{tabular}{|ll|}
\hline Networks & Functions \\
$\square$ Co-expression & G2/M transition of mitotic cell cycle \\
$\square$ Physical interactions & G1/S transition of mitotic cell cycle \\
Shared protein domains & Cell cycle G2/M phase transition \\
Predicted & Ras protein signal transduction \\
Pathway & Cell cycle checkpoint \\
Co-localization & \\
\hline
\end{tabular}

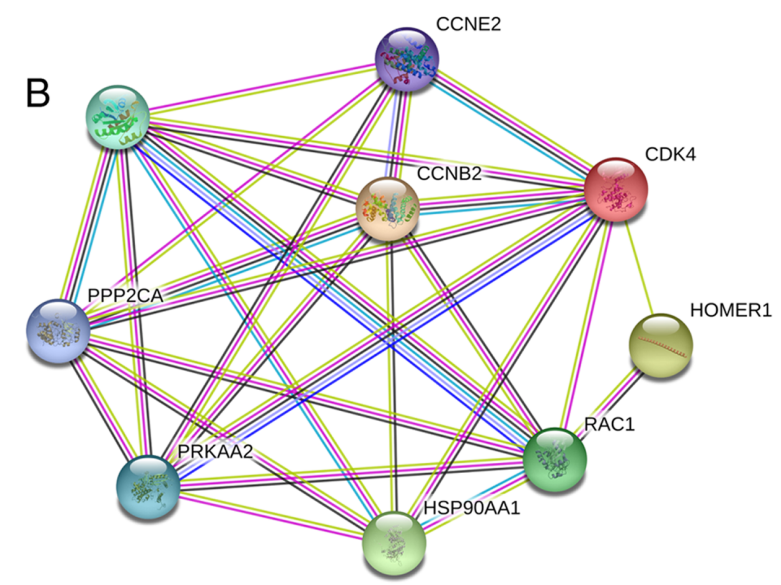

$$
\begin{aligned}
& \begin{array}{l}
\text { Known interactions Predicted interactions Others } \\
- \text { From curated databases }- \text { Gene co-occurrence } \\
- \text { Textmining }
\end{array} \\
& \text { - Experimentally determined _Co-expression } \\
& \text {-P Protein homology }
\end{aligned}
$$

Figure 10. Protein-protein and gene-gene interaction networks among CCNB2, CCNE2, CDK4, HOMER1, HSP90AA1, NRAS, PPP2CA, PRKAA2 and RAC1. (A) Gene-gene interaction network of these 9 genes obtained from GeneMANIA. (B) Protein-protein interaction network of these 9 genes obtained from STRING. 


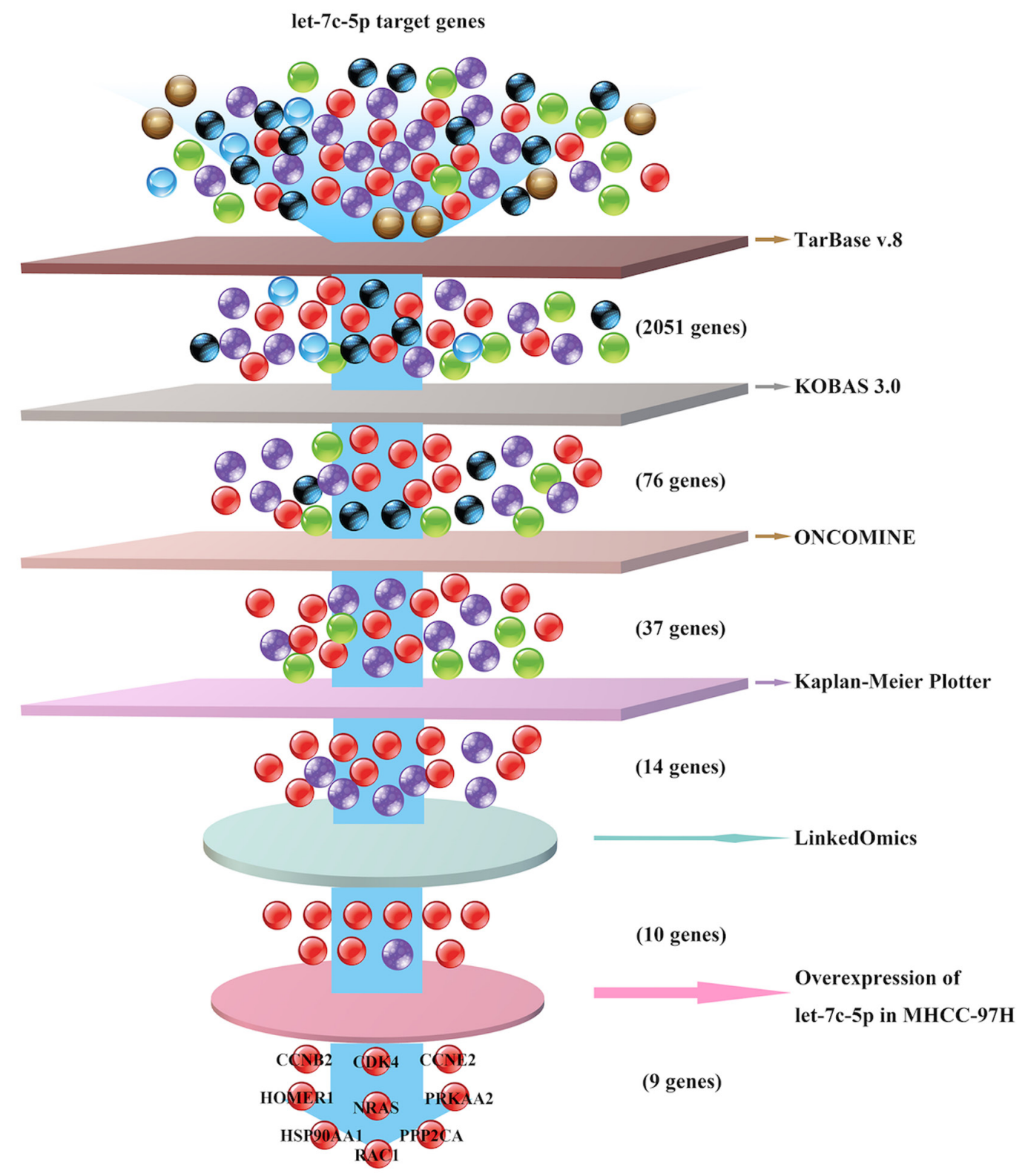

Figure 11. Analytical methodology used to refine let-7c target genes in liver hepatocellular carcinoma.

and maintenance of tumor phenotypes in LIHC (68). NRAS is a proto-oncogene that is associated with several human cancer types, due to its activating mutation (69) demonstrated marked upregulation of wild-type NRAS in LIHC cell lines and patient tissues, whereas NRAS expression is associated with poor patient survival (70). RAC1 is a member of the Rac subfamily of GTPases and serves an important role in several cellular processes, including cell proliferation, cytoskeletal recombination, antimicrobial cytotoxicity and epithelial differentiation (71). It has been demonstrated that RAC1 serves an important role in LIHC progression and metastasis $(72,73)$. Knockdown of RAC1 suppresses the expansion of LIHC cells (74). These results suggested that let-7c served an important role, not only in the regulation of the cell cycle, but also in phenotype progression and metastasis of LIHC. Correlation analysis between let-7c and its target genes, as well as the in vitro cell experiments, indicated that let-7c regulated their expression in LIHC. Therefore, low expression levels of let-7c in LIHC may be considered to be an important factor leading to the high expression of its target genes. The mechanism of action of let-7c may influence the formation and development of LIHC via the PI3K/Akt and FoxO signaling pathways. This may be a possible explanation for the negative association observed between let-7c expression and poor LIHC prognosis.

In conclusion, the present study demonstrated that let-7c may be considered to be an anti-oncogene of LIHC, which mainly affects the cell cycle of LIHC cells via the PI3K-Akt and FoxO signaling pathways. Among its target genes, CCNE2 may be a promising candidate biomarker for disease and poor prognosis in LIHC. The increase in the expression levels of let-7c may be an effective way to improve the prognosis, survival rate and treatment of LIHC. The present study provided novel insights into the mechanisms of LIHC occurrence and development and identified let-7c as a promising therapeutic target for use in the treatment of LIHC. 


\section{Acknowledgements}

Not applicable.

\section{Funding}

No funding was received.

\section{Availability of data and materials}

The data that support the findings of this study are available from the public databases mentioned in this article but restrictions apply to the availability of these data, which were used under license for the current study, and so are not publicly available. Data are however available from the authors upon reasonable request and with permission of the public databases mentioned in this article. The PCR data generated in the present study are available from the corresponding author upon reasonable request.

\section{Authors' contributions}

YL and PL designed the present study. PL and NW performed the experiments. YL, PL and NW analyzed the data and prepared the figures. YL drafted the initial manuscript. All authors have read and approved the final manuscript.

\section{Ethics approval and consent to participate}

Not applicable.

\section{Patient consent for publication}

Not applicable.

\section{Competing interests}

The authors declare that they have no competing interests.

\section{References}

1. Bray F, Ferlay J, Soerjomataram I, Siegel RL, Torre LA and Jemal A: Global cancer statistics 2018: GLOBOCAN estimates of incidence and mortality worldwide for 36 cancers in 185 countries. CA Cancer J Clin 68: 394-424, 2018.

2. Siegel RL, Miller KD and Jemal A: Cancer statistics, 2018. CA Cancer J Clin 68: 7-30, 2018

3. Kim NG, Nguyen PP, Dang H, Kumari R, Garcia G, Esquivel CO and Nguyen MH: Temporal trends in disease presentation and survival of patients with hepatocellular carcinoma: A real-world experience from 1998 to 2015. Cancer 124: 2588-2598, 2018.

4. Krol J, Loedige I and Filipowicz W: The widespread regulation of microRNA biogenesis, function and decay. Nat Rev Genet 11: 597-610, 2010.

5. Huntzinger E and Izaurralde E: Gene silencing by microRNAs: Contributions of translational repression and mRNA decay. Nat Rev Genet 12: 99-110, 2011.

6. Inui M, Martello G and Piccolo S: MicroRNA control of signal transduction. Nat Rev Mol Cell Biol 11: 252-263, 2010.

7. Friedman RC, Farh KK, Burge CB and Bartel DP: Most mammalian mRNAs are conserved targets of microRNAs. Genome Res 19: 92-105, 2009

8. Sharma S, Kelly TK and Jones PA: Epigenetics in cancer. Carcinogenesis 31: 27-36, 2010.

9. Ryan BM, Robles AI and Harris CC: Genetic variation in microRNA networks: The implications for cancer research. Nat Rev Cancer 10: 389-402, 2010.
10. Huang $\mathrm{S}$ and He X: The role of microRNAs in liver cancer progression. Br J Cancer 104: 235-240, 2011.

11. Gailhouste L and Ochiya T: Cancer-related microRNAs and their role as tumor suppressors and oncogenes in hepatocellular carcinoma. Histol Histopathol 28: 437-451, 2013.

12. Li D, Zhang J and Li J: Role of miRNA sponges in hepatocellular carcinoma. Clin Chim Acta 500: 10-19, 2020.

13. Wang J, Lu L, Luo Z, Li W, Lu Y, Tang Q and Pu J: miR-383 inhibits cell growth and promotes cell apoptosis in hepatocellular carcinoma by targeting IL-17 via STAT3 signaling pathway. Biomed Pharmacother 120: 109551, 2019.

14. Au SL, Wong CC, Lee JM, Fan DN, Tsang FH, Ng IO and Wong CM: Enhancer of zeste homolog 2 epigenetically silences multiple tumor suppressor microRNAs to promote liver cancer metastasis. Hepatology 56: 622-631, 2012

15. Zhu X, Wu L, Yao J, Jiang H, Wang Q, Yang Z and Wu F: MicroRNA let-7c inhibits cell proliferation and induces cell cycle arrest by targeting CDC25A in human hepatocellular carcinoma. PLoS One 10: e0124266, 2015.

16. Shimizu S, Takehara T, Hikita H, Kodama T, Miyagi T, Hosui A, Tatsumi T, Ishida H, Noda T, Nagano H, et al: The let-7 family of microRNAs inhibits Bcl-xL expression and potentiates sorafenib-induced apoptosis in human hepatocellular carcinoma. J Hepatol 52: 698-704, 2010.

17. Martini M, De Santis MC, Braccini L, Gulluni F and Hirsch E: PI3K/AKT signaling pathway and cancer: An updated review. Ann Med 46: 372-383, 2014.

18. Guo H, German P, Bai S, Barnes S, Guo W, Qi X, Lou H, Liang J, Jonasch E, Mills GB and Ding Z: The PI3K/AKT Pathway and renal cell carcinoma. J Genet Genomics 42: 343-353, 2015.

19. Faes $\mathrm{S}$ and Dormond O: PI3K and AKT: Unfaithful partners in cancer. Int J Mol Sci 16: 21138-21152, 2015.

20. Zhou Q, Lui VW and Yeo W: Targeting the PI3K/Akt/mTOR pathway in hepatocellular carcinoma. Future Oncol 7: 1149-1167, 2011.

21. Hou YQ, Yao Y, Bao YL, Song ZB, Yang C, Gao XL, Zhang WJ, Sun LG, Yu CL, Huang YX, et al: Juglanthraquinone C induces intracellular ROS increase and apoptosis by activating the Akt/Foxo signal pathway in HCC cells. Oxid Med Cell Longev 2016: 4941623,2016.

22. Wang Q, Yu WN, Chen X, Peng XD, Jeon SM, Birnbaum MJ, Guzman G and Hay N: Spontaneous hepatocellular carcinoma after the combined deletion of Akt isoforms. Cancer Cell 29: 523-535, 2016.

23. Deng M, Bragelmann J, Schultze JL and Perner S: Web-TCGA: An online platform for integrated analysis of molecular cancer data sets. BMC Bioinformatics 17: 72, 2016.

24. Ma Z, Liu T, Huang W, Liu H, Zhang HM, Li Q, Chen Z and Guo AY: MicroRNA regulatory pathway analysis identifies miR-142-5p as a negative regulator of TGF- $\beta$ pathway via targeting SMAD3. Oncotarget 7: 71504-71513, 2016.

25. Wong NW, Chen Y, Chen S and Wang X: OncomiR: An online resource for exploring pan-cancer microRNA dysregulation. Bioinformatics 34: 713-715, 2018.

26. Karagkouni D, Paraskevopoulou MD, Chatzopoulos S, Vlachos IS, Tastsoglou S, Kanellos I, Papadimitriou D, Kavakiotis I, Maniou S, Skoufos G, et al: DIANA-TarBase v8: A decade-long collection of experimentally supported miRNA-gene interactions. Nucleic Acids Res 46: D239-D245, 2018.

27. Kanehisa M and Goto S: KEGG: Kyoto encyclopedia of genes and genomes. Nucleic Acids Res 28: 27-30, 2000.

28. Ai C and Kong L: CGPS: A machine learning-based approach integrating multiple gene set analysis tools for better prioritization of biologically relevant pathways. J Genet Genomics 45: 489-504, 2018

29. Xie C, Mao X, Huang J, Ding Y, Wu J, Dong S, Kong L, Gao G, Li CY and Wei L: KOBAS 2.0: A web server for annotation and identification of enriched pathways and diseases. Nucleic Acids Res 39: W316-W322, 2011.

30. Wu J, Mao X, Cai T, Luo J and Wei L: KOBAS server: A web-based platform for automated annotation and pathway identification. Nucleic Acids Res 34: W720-W724, 2006.

31. Rhodes DR, Yu J, Shanker K, Deshpande N, Varambally R, Ghosh D, Barrette T, Pandey A and Chinnaiyan AM: ONCOMINE: A cancer microarray database and integrated data-mining platform. Neoplasia 6: 1-6, 2004.

32. Edgar R, Domrachev M and Lash AE: Gene expression omnibus: NCBI gene expression and hybridization array data repository. Nucleic Acids Res 30: 207-210, 2002.

33. von Eschenbach $\mathrm{AC}$ and Buetow $\mathrm{K}$ : Cancer informatics vision: CaBIG. Cancer Inform 2: 22-24, 2007. 
34. Menyhárt O, Nagy Á and Győrffy B: Determining consistent prognostic biomarkers of overall survival and vascular invasion in hepatocellular carcinoma. R Soc Open Sci 5: 181006, 2018.

35. Nagy A, Lanczky A, Menyhart O and Gyorffy B: Validation of miRNA prognostic power in hepatocellular carcinoma using expression data of independent datasets. Sci Rep 8: 9227, 2018.

36. Vasaikar SV, Straub P, Wang J and Zhang B: LinkedOmics: Analyzing multi-omics data within and across 32 cancer types Nucleic Acids Res 46: D956-D963, 2018.

37. Chandrashekar DS, Bashel B, Balasubramanya SAH, Creighton CJ, Ponce-Rodriguez I, Chakravarthi BVSK and Varambally S: UALCAN: A portal for facilitating tumor subgroup gene expression and survival analyses. Neoplasia 19: 649-658, 2017

38. Gao J, Aksoy BA, Dogrusoz U, Dresdner G, Gross B, Sumer SO, Sun Y, Jacobsen A, Sinha R, Larsson E, et al: Integrative analysis of complex cancer genomics and clinical profiles using the cBioPortal. Sci Signal 6: pl1, 2013.

39. Cerami E, Gao J, Dogrusoz U, Gross BE, Sumer SO, Aksoy BA, Jacobsen A, Byrne CJ, Heuer ML, Larsson E, et al: The cBio cancer genomics portal: An open platform for exploring multidimensional cancer genomics data. Cancer Discov 2: 401-404, 2012.

40. Livak KJ and Schmittgen TD: Analysis of relative gene expression data using real-time quantitative PCR and the 2(-Delta Delta C(T)) method. Methods 25: 402-408, 2001.

41. Ashburner M, Ball CA, Blake JA, Botstein D, Butler H, Cherry JM, Davis AP, Dolinski K, Dwight SS, Eppig JT, et al: Gene ontology: Tool for the unification of biology. The Gene Ontology Consortium. Nat Genet 25: 25-29, 2000.

42. The Gene Ontology C: The Gene Ontology Resource: 20 years and still GOing strong. Nucleic Acids Res 47: D330-D338, 2019.

43. Reimand J, Kull M, Peterson H, Hansen J and Vilo J: g:Profiler-a web-based toolset for functional profiling of gene lists from large-scale experiments. Nucleic Acids Res 35: W193-W200, 2007.

44. Raudvere U, Kolberg L, Kuzmin I, Arak T, Adler P, Peterson H and Vilo J: g:Profiler: A web server for functional enrichment analysis and conversions of gene lists (2019 update). Nucleic Acids Res 47: W191-W198, 2019.

45. Warde-Farley D, Donaldson SL, Comes O, Zuberi K, Badrawi R, Chao P, Franz M, Grouios C, Kazi F, Lopes CT, et al: The GeneMANIA prediction server: Biological network integration for gene prioritization and predicting gene function. Nucleic Acids Res 38: W214-W220, 2010.

46. Max F, Rodriguez H, Lopes C, Zuberi K, Montojo J, Bader GD and Morris Q: GeneMANIA update 2018. Nuclc Acids Res 46: W60-W64, 2018.

47. Szklarczyk D, Franceschini A, Wyder S, Forslund K, Heller D, Huerta-Cepas J, Simonovic M, Roth A, Santos A, Tsafou KP, et al: STRING v10: Protein-protein interaction networks, integrated over the tree of life. Nucleic Acids Res 43 (Database Issue): D447-D452, 2015

48. Szklarczyk D, Gable AL, Lyon D, Junge A, Wyder S, Huerta-Cepas J, Simonovic M, Doncheva NT, Morris JH, Bork P, et al: STRING v11: Protein-protein association networks with increased coverage, supporting functional discovery in genome-wide experimental datasets. Nucleic Acids Res 47: D607-D613, 2019.

49. Song Y, Wang F, Huang Q, Cao Y, Zhao Y and Yang C: MicroRNAs contribute to hepatocellular carcinoma. Mini Rev Med Chem 15: 459-466, 2015.

50. Han Y, Liu Y, Fu X, Zhang Q, Huang H, Zhang C, Li W and Zhang J: miR-9 inhibits the metastatic ability of hepatocellular carcinoma via targeting beta galactoside alpha-2,6-sialyltransferase 1. J Physiol Biochem 74: 491-501, 2018.

51. Wang Y, Tai Q, Zhang J, Kang J, Gao F, Zhong F, Cai L, Fang F and Gao Y: MiRNA-206 inhibits hepatocellular carcinoma cell proliferation and migration but promotes apoptosis by modulating cMET expression. Acta Biochim Biophys Sin (Shanghai) 51: 243-253, 2019.

52. Zhu XM, Wu LJ, Xu J, Yang R and Wu FS: Let-7c microRNA expression and clinical significance in hepatocellular carcinoma. J Int Med Res 39: 2323-2329, 2011.

53. Jilek JL, Zhang QY, Tu MJ, Ho PY, Duan Z, Qiu JX and Yu AM: Bioengineered Let-7c inhibits orthotopic hepatocellular carcinoma and improves overall survival with minimal immunogenicity. Mol Ther Nucleic Acids 14: 498-508, 2019.

54. Luo H, Hao E, Tan D, Wei W, Xie J, Feng X, Du Z, Huang C, Bai G, Hou Y, et al: Apoptosis effect of Aegiceras corniculatum on human colorectal cancer via activation of FoxO signaling pathway. Food Chem Toxicol 134: 110861, 2019.
55. Farhan M, Wang H, Gaur U, Little PJ, Xu J and Zheng W: FOXO signaling pathways as therapeutic targets in cancer. Int J Biol Sci 13: 815-827, 2017.

56. Nam HJ and van Deursen JM: Cyclin B2 and p53 control proper timing of centrosome separation. Nat Cell Biol 16: 538-549, 2014

57. Zhang Q, Sun S, Zhu C, Zheng Y, Cai Q, Liang X, Xie H and Zhou J: Prediction and analysis of weighted genes in hepatocellular carcinoma using bioinformatics analysis. Mol Med Rep 19: 2479-2488, 2019.

58. Lauper N, Beck AR, Cariou S, Richman L, Hofmann K, Reith W, Slingerland JM and Amati B: Cyclin E2: A novel CDK2 partner in the late $\mathrm{G} 1$ and $\mathrm{S}$ phases of the mammalian cell cycle. Oncogene 17: 2637-2643, 1998

59. Hwang HC and Clurman BE: Cyclin E in normal and neoplastic cell cycles. Oncogene 24: 2776-2786, 2005.

60. Caldon CE and Musgrove EA: Distinct and redundant functions of cyclin E1 and cyclin E2 in development and cancer. Cell Div 5: 2,2010

61. Sherr CJ, Beach D and Shapiro GI: Targeting CDK4 and CDK6 From discovery to therapy. Cancer Discov 6: 353-367, 2016.

62. Xiang X, You XM and Li LQ: Expression of HSP90AA1/HSPA8 in hepatocellular carcinoma patients with depression. Onco Targets Ther 11: 3013-3023, 2018.

63. Piredda ML, Gaur G, Catalano G, Divona M, Banella C, Travaglini S, Puzzangara MC, Voso MT, Lo-Coco F and Noguera NI: PML/RARA inhibits expression of HSP90 and its target AKT. Br J Haematol 184: 937-948, 2019.

64. Hou C, Li Y, Liu H, Dang M, Qin G, Zhang N and Chen R: Profiling the interactome of protein kinase $\mathrm{C}$ zeta by proteomics and bioinformatics. Proteome Sci 16: 5, 2018

65. Fox MM, Phoenix KN, Kopsiaftis SG and Claffey KP: AMP-activated protein kinase alpha 2 isoform suppression in primary breast cancer alters AMPK growth control and apoptotic signaling. Genes Cancer 4: 3-14, 2013.

66. Vila IK, Yao Y, Kim G, Xia W, Kim H, Kim SJ, Park MK, Hwang JP, González-Billalabeitia E, Hung MC, et al: A UBE2O-AMPK $\alpha 2$ axis that promotes tumor initiation and progression offers opportunities for therapy. Cancer Cell 31: 208-224, 2017.

67. Jardin I, Albarran L, Bermejo N, Salido GM and Rosado JA: Homers regulate calcium entry and aggregation in human platelets: A role for Homers in the association between STIM1 and Orai1. Biochem J 445: 29-38, 2012.

68. Moccia F, Zuccolo E, Poletto V, Turin I, Guerra G, Pedrazzoli P, Rosti V, Porta C and Montagna D: Targeting stim and orai proteins as an alternative approach in anticancer therapy. Curr Med Chem 23: 3450-3480, 2016.

69. Rajalingam K, Schreck R, Rapp UR and Albert S: Ras oncogenes and their downstream targets. Biochim Biophys Acta 1773: 1177-1195, 2007

70. Dietrich P, Gaza A, Wormser L, Fritz V, Hellerbrand C and Bosserhoff AK: Neuroblastoma RAS viral oncogene homolog (NRAS) is a novel prognostic marker and contributes to sorafenib resistance in hepatocellular carcinoma. Neoplasia 21: 257-268,2019.

71. Xiang RF, Stack D, Huston SM, Li SS, Ogbomo H, Kyei SK and Mody CH: Ras-related C3 botulinum toxin substrate (Rac) and Src Family Kinases (SFK) Are proximal and essential for phosphatidylinositol 3-Kinase (PI3K) activation in natural Killer (NK) Cell-mediated Direct Cytotoxicity against cryptococcus neoformans. J Biol Chem 291: 6912-6922, 2016.

72. Chen L, Chan TH, Yuan YF, Hu L, Huang J, Ma S, Wang J, Dong SS, Tang KH, Xie D, et al: CHD1L promotes hepatocellular carcinoma progression and metastasis in mice and is associated with these processes in human patients. J Clin Invest 120: $1178-1191,2010$

73. Zhao P, Zhang W, Wang SJ, Yu XL, Tang J, Huang W, Li Y, Cui HY, Guo YS, Tavernier J, et al: HAb18G/CD147 promotes cell motility by regulating annexin II-activated RhoA and Rac1 signaling pathways in hepatocellular carcinoma cells. Hepatology 54: 2012-2024, 2011.

74. Ran RZ, Chen J, Cui LJ, Lin XL, Fan MM, Cong ZZ, Zhang H, Tan WF, Zhang GQ and Zhang YJ: miR-194 inhibits liver cancer stem cell expansion by regulating RAC1 pathway. Exp Cell Res 378: 66-75, 2019.

This work is licensed under a Creative Commons Attribution-NonCommercial-NoDerivatives 4.0 International (CC BY-NC-ND 4.0) License. 